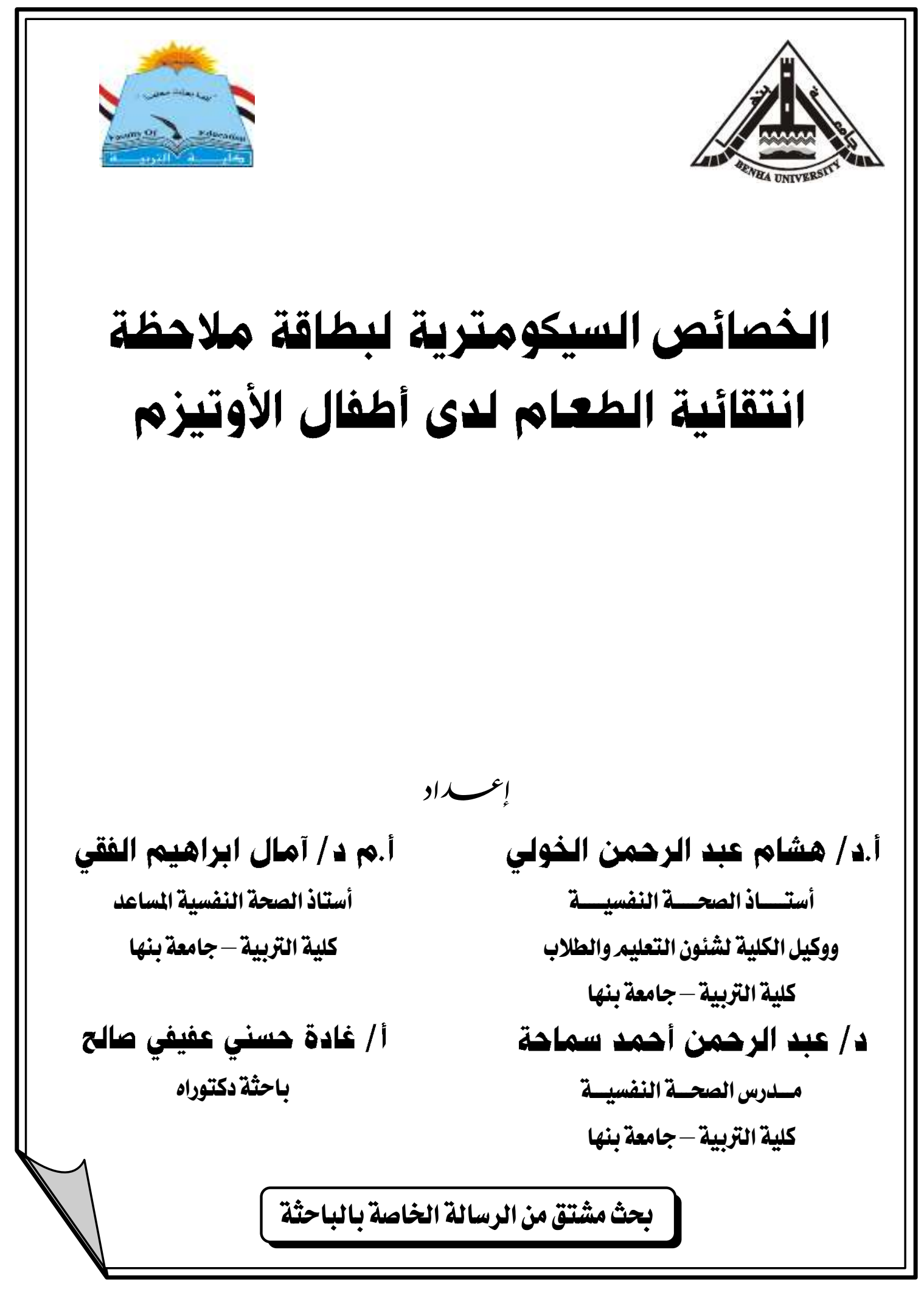




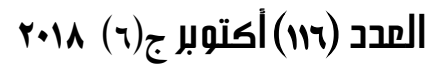

مجلة كلية التربية ببنها

الخصائص السيكومترية لبطاقة ملاحظة انتقائية الطعام لاى أطفال الأوتيزم

! إ

أ.هم د / آمال ابراهيم الفتقي

أستاذ الصحة النفسية المساعل الماند

كلية التربية - جامعة بنها

غادة حسني عفيفي صالح

باحثة دكتوراه
أ. دـ / هشام عبد الرحمن الخولي

أستــــاذ الصحســة النفسيــــة

ووكيل الكلية لشئون التعليموالطلاب النياب

كلية التربية- - جامعة بنها

هـ / عبد الرحمن أحمد سماحة

مسلدرس الصحسة النفسيـة

\section{ملذص البحثث}

هدفت الدراسة إلى اعداد بطاقة ملاحظة انتقائية الطعام لاى أطفال الأوتيزم، وللتحقق

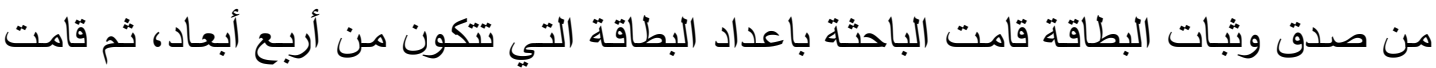

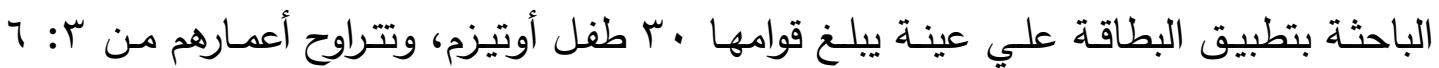

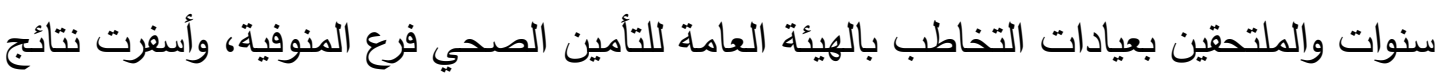

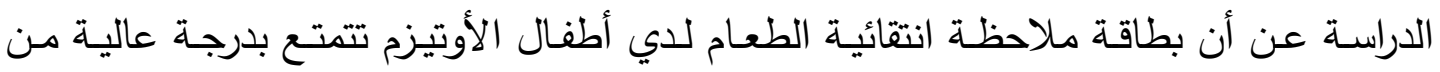

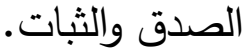




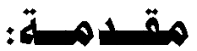

يُعد الأوتيزم أحد الاضطرابات النمائية العصبية الشاملة التي تعتبر من أكثر مشكلات الطفولة إزعاجًا وإرباكًا وحيرة لأنها تتضمن اضطرابًا في جوانب الآداء النفسي خـلال مرحلة

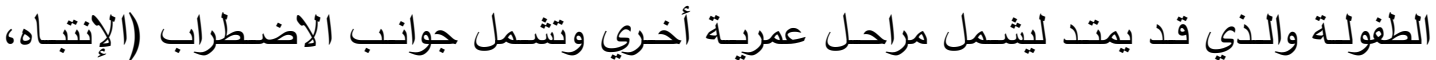
والإدراك، والتعلم، واللغة، ومهارات التواصل، والمهارات الحسية، والحركية) وكل ذلك ينعكس سلبًا على كل من يتعامل مـع هؤلاء الأطفال من أسر ومعلمين وإختصاصيين وأقران، كما ينعكس سلبًا أيضًا على الطفل حيث أن كل هؤلاء لا يعرفون كيفية التعامل مـع مثل هؤلاء الاهل

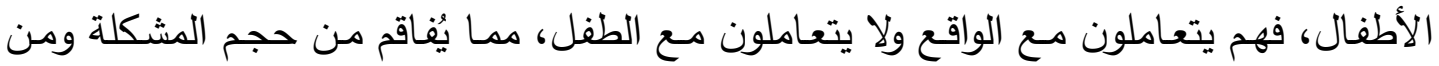

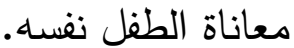

ويتميز الأوتيزم بالقصور المستمر في التواصل والتفاعل الاجتماعي المتبادل وذلك في العديد مـن السياقات، بالإضـافة إلى وجود نمـاذج محددة وذات طـابع آلي تكـراري للسـلوك

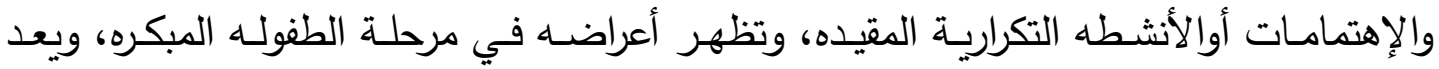

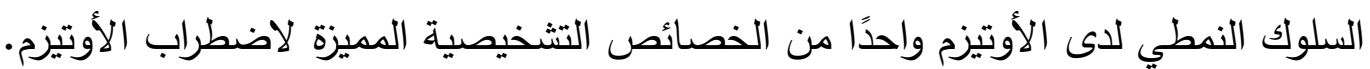

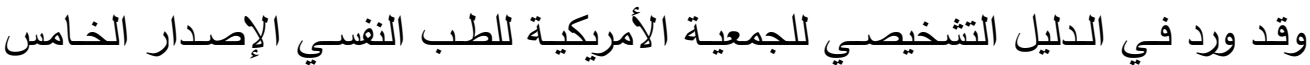
(American Psychiatric Association, 2013:50) النمطية تتباين، حيث يمكن أن تكون لفظية أوغير لفظية (علي سبيل المثال: الصداء اللفظي

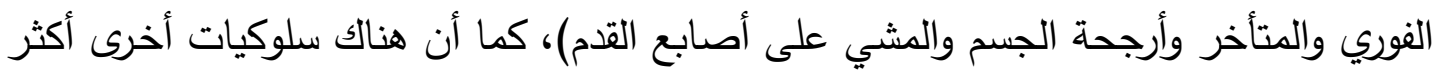
تعقيدًا وتتمثل في الإصرار على تماثل الأشياء، والإلتزام غير المرن بالروتين، وأنماط أشبه الفيه

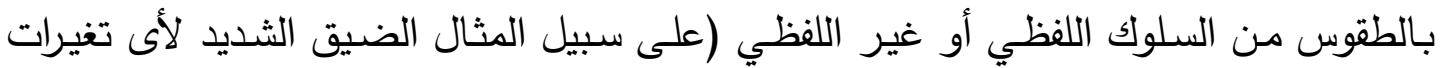

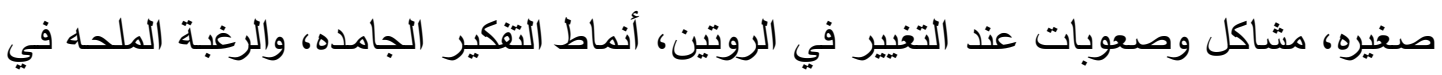
أكل نفس الطعام يوميًا أوالمشي من نفس الطريق يوميًا). وتُعد انتقائية الطعام بمثابة شكل من أشكال السلوكيات النمطية، بالإضـافة إلى أنها إحدى مشكلات الأكل والتغذيـة والتي يعاني منها نسبة ليست بالقليلة من الأوتيزم، كمـا أن النان التناول المحدود من الأطعمة يؤدي إلى عدم الكفاية الغذائية ممـا يسبب خطرًا على الصحة

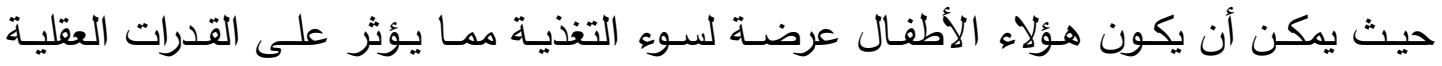
والسلوكية، كما يؤثر سلبًا على صحة العظام وعلى الصحة الجسدية، وعلى جودة الحيـاة

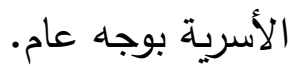




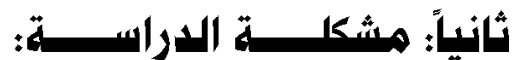

أوضح سواريز (2014:425, Suarez) أن درجة إنتقائية الطعام لدى الأوتيزم يمكن أن تتراوح ما بين الدرجة الثديدة حيث يتم الاعتماد في تغذية الطفل علي الأنبوب كمصدر بديل

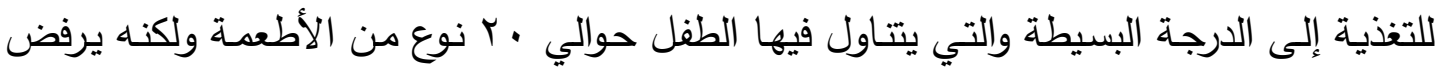
أنواع أخري من الأطعمة مما يعرض الطفل إلى أوجه القصور في بعض العناصر الغذائية. ولقد أشارت نتائج المقابلة الاستطلاعية الحرة التي قامت بها الباحثة مع بعض أمهات

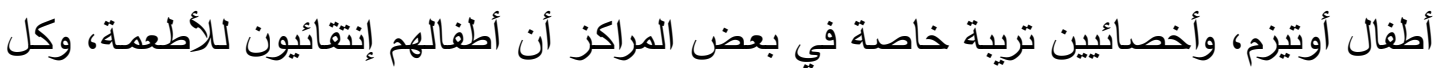

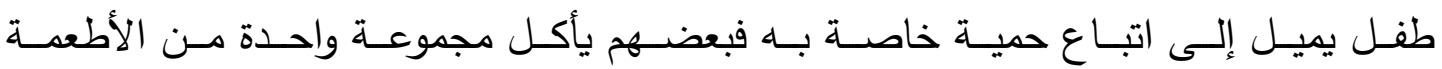

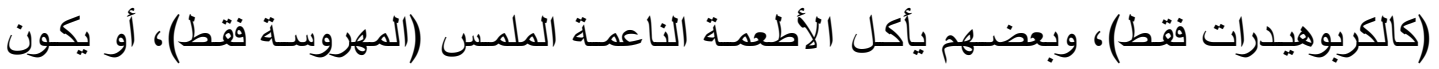

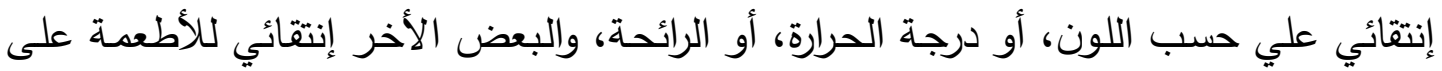

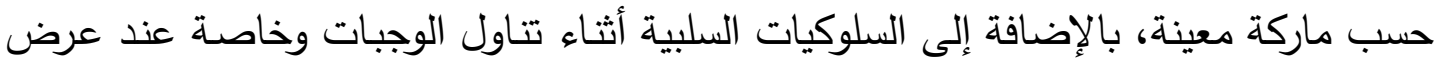

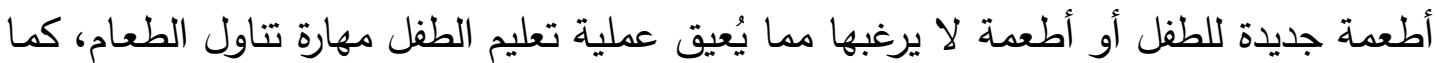
يعيق تكيف الطفل الاجتماعي فهوسلوك غير مقبول من الآخرين. وقد أكد كل من سواريز، وكرينيون (Suarez \& Crinion., 2015)، ويليامز ودالريمبل، ونيل (Williams, Dalrymple, Neal., 2000) على أن انتقائية الطعام شائعة لاى الكثير من أطفال الأوتيزم ويرتبط بها سلوكيات تتاول الطعام غير الملائمة نتيجة لتقديم

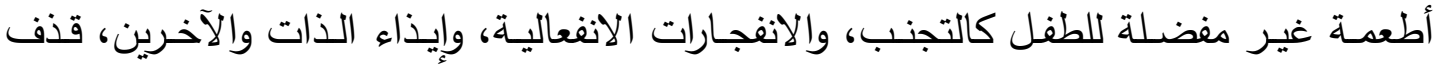

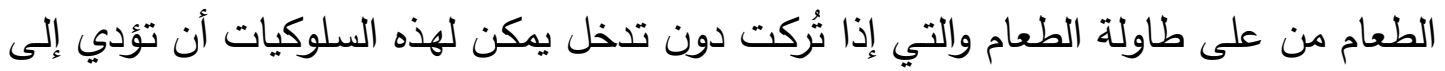
إجهاد وتوتروقلق وشعور بالذنب والعديد من الشككلات الصحية التي تصيب الوالدين فهم يجدون صعوبة في تناول الطعام بسبب المشكلات السلوكية التي يظهرها أطفالهم، مما يجعل الآبـاء لا يقدمون على حضور المناسبات الإجتماعيـة اوالذهاب إلى الأمـاكن العامـة (أعياد

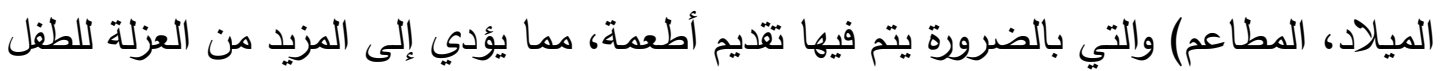

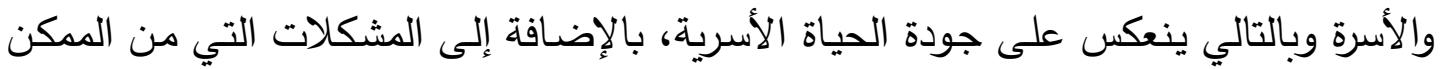

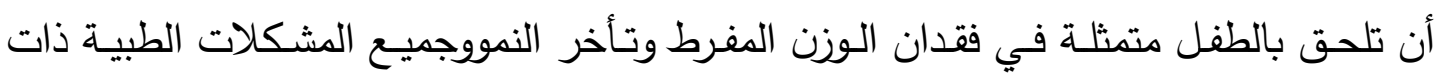
الصلة بالناحية الغذائية والضعف الإجتماعي والقصور المعرفي والآداء الأكاديمي. 
ومما يُزيد من حجم المشكلة أن بعض الآباء قد يُّدمون لأطفالهم طعامهم المُفضل على اعتبار أنه طعام أيضًا، وأن تتاول أي طعام أفضل من لاشيء، ويلجأون إلى طعام الرضع وتقديم الطعام مهروس وقد يلجأون إلى الككملات الغذائية وفاتح الثهية وذلك بدون استثارة الطبيب. كما أشارت بعض الأمهات خلال المقابلة الاستطلاعية أنه عند فحص طبيب الأطفال لأبنائهم تشير نتائج الفحص أن وزن وطول الطفل مناسب لسنه، مما يجعل الآباء لا يشعرون بالمشكلة وتركه يتتاول مـا يُفضـله فقط وتسليمهم بالأمر الواقع وفقدان الأمسل في أن يتتاول

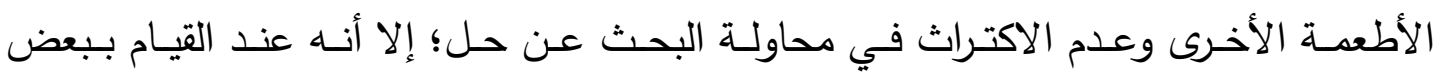
الفحوصات والتي توضح غالبًا أن هنالك نقص في الفيتاميات والمعادن. ان الأهل غالبًا ما يتلقون معلومات خاطئة من المحيطين وأيضًا غير متخصصين ويوصون الآباء بعدم الانزعاج والثعور بالقلق حيال مشكلة انتقائية الطعام وأنه سيتخطي هذه المشكلة إن عاجلاً أم آجلاً وأنه سيأكل حينما يشعر بجوعِ كاف أويوصون بـون الآباء بتجويع الطفل

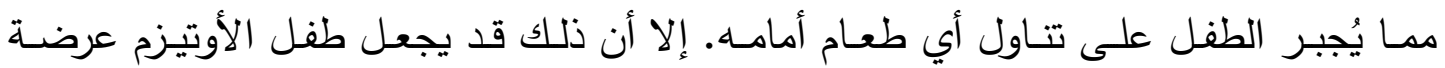
لمخاطر شديدة صحيًا، واجتماعيًا، وأكاديميًا......

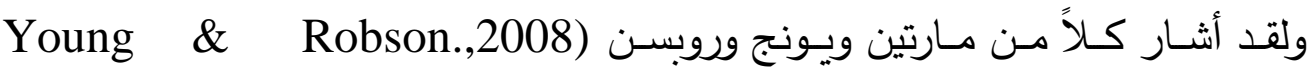
Provost, Crowe بروفوست، وكرو، وأوسبورن، ومكليان، وسكيير (Martins, بضرورة وضع انتقائية الطعام وما يرتبط بها من (Osbourn, McClain Skipper., 2010) سلوكيات سلبية في الإعتبار عند تشخيص الأوتيزم. وبذلك ويمكن تحديد مشكلة الدراسة في التساؤل الآتي:

هل يمكن تقييم وتثخيص انتقائية الطعام لاى أطفال الأوتيزم من خلال اعداد بطاقة ملاحظة انتقائية الطعام وتتمتع بدرجة عالية من الصدق والثبات؟

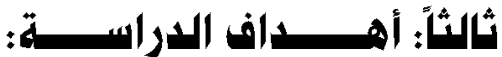

هدفت الدراسـة الحالية إلى اعداد بطاقة ملاحظة انتقائية الطعام لاى اطفال الأوتيزم وتتمتع بدرجة عالية من الصدق والثبات. 


\section{رابعاً: أهميسيسة الدراسيستة:}

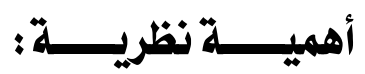

تعتبر الدراسة العربية الأولى في حدود علم الباحثة- التي تناولت انتقائية الطعام لدى

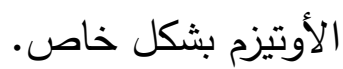
• تم تتاول العلاقة بين انتقائية الطعام وقصور المعالجة الحسية لدى الأوتيزم.

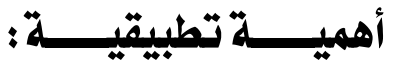

" محاولـة لمسـاعدة الأخصـائيين والأطبـاء وبخاصـة أطباء الأطفال والنفسية والعصبية

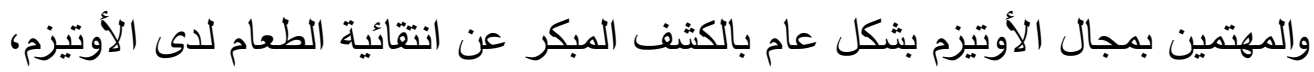
والبحث عن الأسباب العضوية أوالبيئية وتصمييم تدخلات سلوكية تستهدف السلوك النمطي وفقًا لوظيفة الاستجابة وليس الثكل التصنيفي فقط. هإن تقديم بطاقة ملاحظة انتقائية الطعام لاى لأطفال أوتيزم بمثابة نقطة انطلاقة للبحث

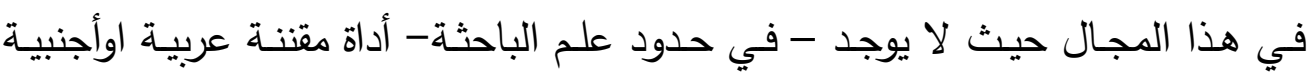

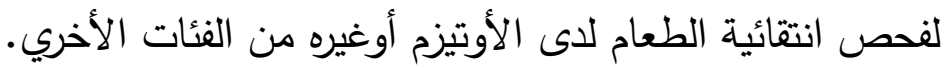

\section{خامساً: مصطلحسات الدراسسية:}

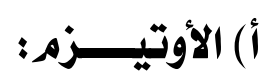

(American Psychiatric Association, عرفته الجمعية الأمريكية للطب النفسي (2013:50: بأنه أحد الإضطرابات النمائيه العصبية، يتميز بالقصور المستمر في التواصل الاجتماعي المتبادل والتفاعل الاجتماعي وذلك في العديد من السياقات، بالإضـافة إلى وجود نماذج محددة ومكررة من السلوك والإهتمامات أوالأنشطه التكرارية المقيده وتظهر أعراضهه في لي

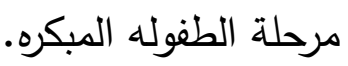

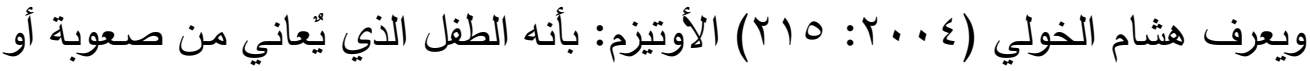
قصور في المهارات الاجتماعية المعرفية والتي تتمثل في مهارات الانتباه، والتفاعل الاجتماعي، والتواصل، والقصور اللغوي، كما يُعاني من سلوكيات نمطية غير مرغوبة، هذا وتظهر هذه الأعراض خلال مرحلة الطفولة المبكرة وقبل ان يتجاوز الطفل عامه الثالث. 
ويُعرف اجرائيًا: بالدرجة التي يحصل عليها الطفل على قائمة المظاهر السلوكية للطفل

$$
\text { الأوتيزم (إعداد/ هشام الخولي، ع . . ب). }
$$

\section{ب) إنتقائية الطعاه للدى الأوتيزم:}

تُعرفه الباحثة بأنها: أحد أثكال السلوكيات النمطية التي يتصف بها الأوتيزم، وتتضمن محدودية أنواع الأطعمة التي يتناولها الطفل، وتتراوح درجة انتقائية الطعام لدى الأوتيزم ما بين الدرجـة الشديدة حيث يتم الاعتمـاد في تغذية الطفل علي الأنبوب كمصدر بديل للتغذية إلى الدرجة البسيطة والتي يتـاول فيها الطفل أقل من · ب نوع من الأطعمـة، وقد يرجع ذلك إلى عوامل عضوية، أوبيئية، أوكلاهما، وما يرتبط بها من سلوكيات سلبية كالهرب من مكان الأكل والصراخ وإلقاء الأطعمة على الأرض عند تقديم أطعمة لا يفضلها الطفل، وتترك انتقائية الطعام آثارًا سلبية على الطفل والأسرة، كما أنها من مشكلات التغذية والأكل الأكثرشيوعًا لدى الأوتيزم. ويُعرف اجرائيًا: بالدرجة التي يحصل عليها الطفل على بطاقة ملاحظة انتقائية الطعام

$$
\text { أطفال الأوتيزم (إعداد /الباحثة). }
$$

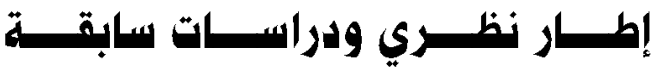

\section{مفهوه انتقائية الطعاه}

يعرف ليدفورد وجيست (Ledford \& Gast, 2006) انتقائية الطعام بأنها محدودية استهلاك الأطعمـة على أسـاس التذوق او الملمس أو الألفة/ المعرفة وهى مشكلة شـائعة لدى الأطفال الذين يعانون من الأوتيزم.

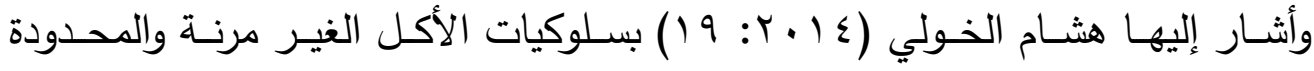
أوروتين صارم في الغذاء حيث يكون لطفل الأوتيزم نظام غذائي وحصري يتألف من أطعمة معينـة قد تكون ذات ملمس أولون أورائحسة معينـة وعندما يحدث تدخل لتوسيع دائرة انتقائيـة الطعام يواجهـ ذلك بسلوكيات عنيفة من قبيل الغضب والعدوان والبكاء والصـراخ والهرب من مكان الأكل والضرب والقاء الأطعمة علي الأرض. كما أشار سواريز (Suarez,2014:1) إلى انتقائية الطعام برفض الطعام علي أساس النوع أواللون أوالتذوق اودرجة الحرارة أوشكل الطعام مما يؤدي إلي نظام غذائي ذاتي مقيد، كما 
أن بعض الأطفال اللذين يعـانون مـن إنتقائيـة الطعـام يتــاولون مـن ه- . 1 مـن الأطعهـة

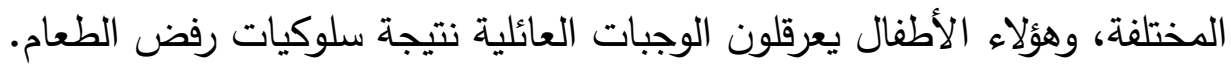

\section{أسباب انتقائية الطعاه للدى الأوتيزه}

ان البحث في أسباب انتقائية الطعام لدى الأوتيزم تُلقي الضوه على طبيعة المشكلة،

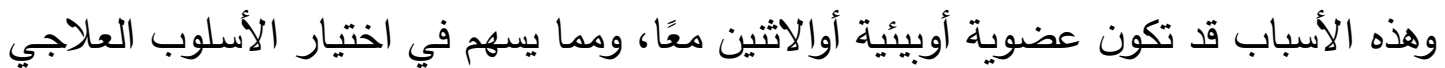
المناسب للحد من انتقائية الطعام. 1-يرجع البعض انتقائية الطعام نتيجة لقصور المعالجة الحسية وهذا مـا اشـار إليه نـادون، وفيلدمان، ودون، وجيزل (Nadon, Feldman, Dunn \& Gisel,2011) على أنه يتضايق الأطفال نتيجة قصور المعالجه الحسيه من كون الطعام ساخنًا جدًا أوباردًا جدًا،

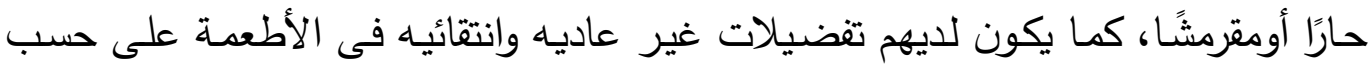

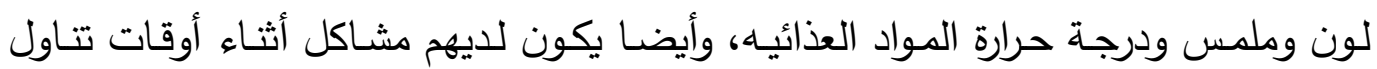

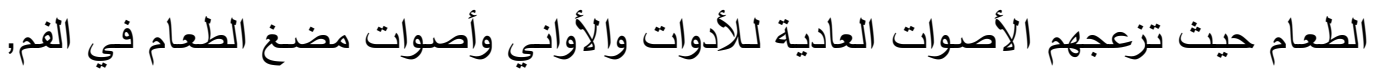

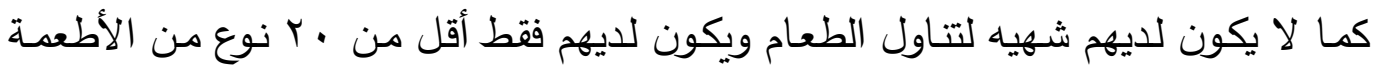
المختلفة في ذخيرتهم الغذائية.

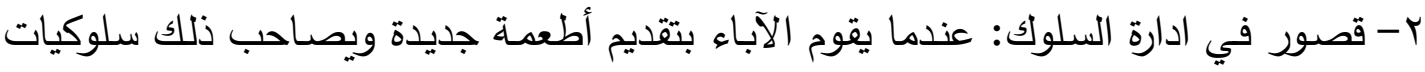

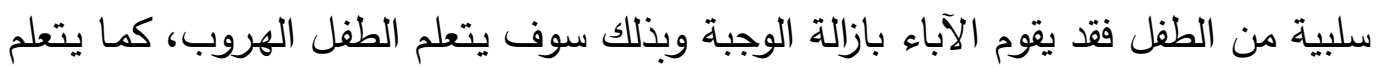

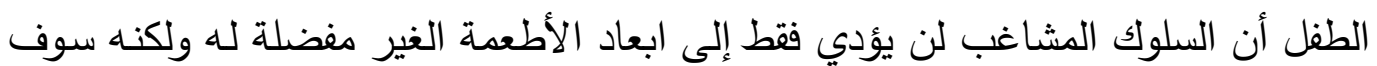
يتعلم ايضًا أنه سيحصل على الأطعمة الدفضلة له، ونتيجة لسلوك الوالدين تتعزز انتقائية الطعام وتستمر (Milnes 2011,8:9).

r- قصور في المهارات الحركية الفمية (مثل ضعف مهارات المضن، وصعوبة تحريك اللسان،

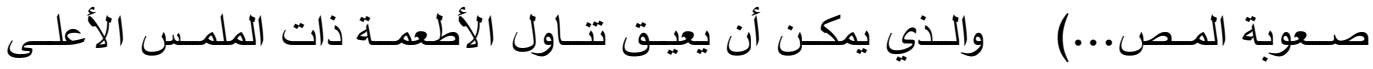
أوالأكثر صـلابة ( Layer, Coleman \& Swartzwelder,2005:89)؛ (Sharp\& Jaquess 2009:169)، وتستطيع معالجة النطق والكلام تقييم الوظيفية الفية الحركية، والوقوف علي المشكلات، ومن ثم التدخل عن طريق الأنشطة العلاجية لتحسن هذه المهارات(Strickland,2009:68 ). 
ع- الأسـر التي تتـاول عدد محدود من الأطعمة كان لديهم أطفال أكثر عرضـة لتـاول عدد

محدود من الأطعمة (انتقائية الطعام) ( Schreck\& William,2005:ro^).

ه- عوامل بيولوجية مثل الخلل الوظيفي العصبي حركي، واضطراب التمثيل الغذائي، وأمراض الجهاز التنفي والقلب، ومشكلات الجهاز الهضمي (2011:8, Milnes)، أوعدم الراحة الجسـية المرتبطـة بالامسـاك، أوالارتجـاع ومـا يصـاحب ذلك من توتر يُسـهم في نقص

الاهتمام بالغذاء (Suarez, Nelson\& Curtis,2012:2).

ففي بعض الحالات قد تكون العوامل البيولوجية هى البداية لسلوكيات رفض الطعام، في

حين ان قصور ادارة السلوك قد تؤدي الى الحفاظ واستمرارية المشكلة (2011:10, Milnes) وتُضيف ستريكلاند (Strickland, 2009) إلى المشكلات الطبية: الآثـار الجانبيـة للـدواء: كأدويـة مضـادات الـذهان والاكتئـاب والوسـواس القهـري وفـرط النشـاط ونقص الانتبـاه، والتي ممكن أن يكـون لهـا آثارهـا الجانبيـة المتمثلـة في تراجـع أوزيـادة الثــية، نقـص أوزيـادة الـوزن، قيء، جفـاف بـالفم، تغيـر فـي حاسـة التـذوق، ألم في البطن، امساك، اسهال. التدخلات الجراحية السـابقة: كعملية تركيب انبوب انفي معدي، مما يجعله ينتابـه الخوف من وضع الأشياء مثل الطعام في فمه. مشاكل الأسنان: كالتسوس، وقرحة الفم، وقرحة أوانتفاخ اللثة. مما يجعل الطفل يلجأ إلى تناول الأطعمة اللينة ويرفض تناول الأطعمة المقرمشة والساخنة والحارة. צ- عمليات الدافعيـة المتمثلة في الثبع والحرمان تلعب دورًا مهمًا في تطوير وتفاقم مشكلات التغذية، ولابد أن تقوم الأبحاث المستقبلية بدور الاستكشاف عن استغلال الثهية ودمج هذا النـوع مـن العمليـة الدافعيـة إلـى التـدخلات والتـي ربمـا يمكـن أن تعـزز مـن فاعليـة العلاج.)(Levin \& Carr, 2001) فقـــ يحـدث الثـبع نتيجـة لشـرب الطفـل أوتتـاول الطعـام بـين الوجبـات يمكن لهـذه الممارسات أن تُضعف التدخلات لعلاج انتقائية الطعام، لأن أي سعرات حرارية اضافية قد يقلل من الدافعية لتناول الطعام أثناء الجلسات \& Seiverling, Williams, Ward-Horner \& Sturmey,2011:493) 


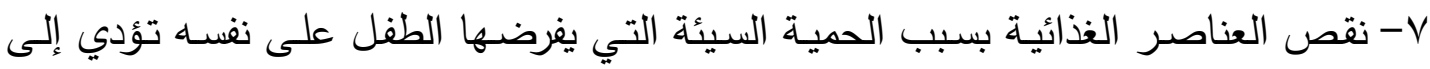

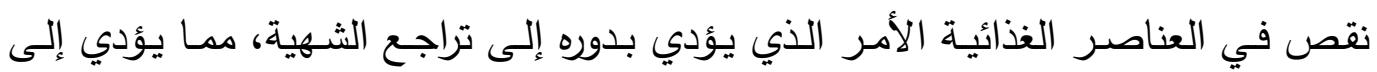
رفض الطعام والعديد من الشكلات السلوكية عند تناول الوجبات، فهي حلقة مُفرغة. (Strickland,2009:68)

ᄉ- خصائص طفل الأوتيزم وما يصاحبه من سلوكيات نمطية من المتمل أن تُبب انتقائية

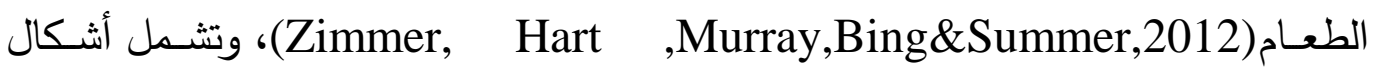
السلوكيات النمطية فيما يتعلق بسلوكيات تتاول الوجبات (الحاجة إلى استخدام نفس الطبق

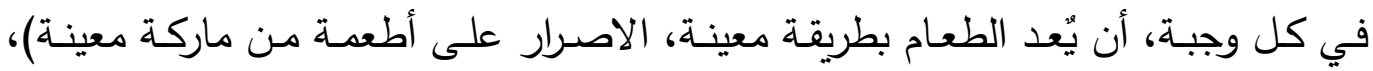

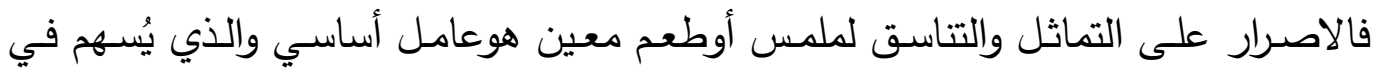
استعداد الطفل لقبول الطفل أطعمـة خاصـة والأطفال اللذين سـلوا درجـات عاليـة علي مقياس السلوكيات النمطية من المحتمل أن يسجلوا مستويات شديدة من انتقائية الطعام .(Suarez, Nelson\& Curtis,2014:925)

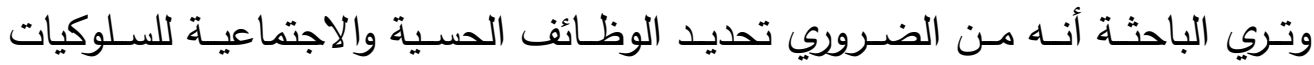
والنمطية، ويجب أن يتت تحديد المداخل العلاجية بناءًا على التنسير الوظيفي للنمطية والذي

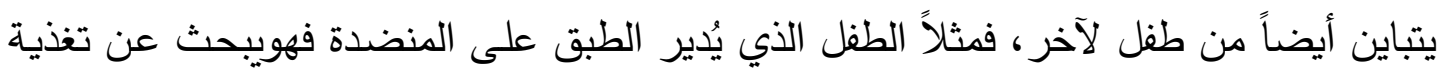

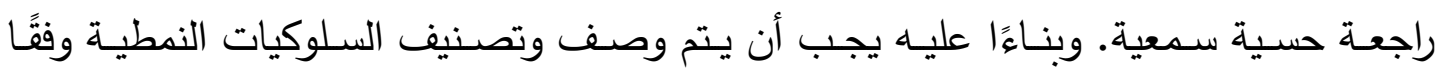
لوظيفتها وليس شكلها. 9- الطفـل الخديج (المولـود قبـل الآوان) والذني يواجـه تحديات طبيـة ويقضـي أسـابيع في المستشفي فور ولادته، ويخضع للعديد من الاجراءات الطبية، ويحتاج لتغذيته أنبوب أنفي معدي والذي قد يسبب الألم الجسدي، ومما قد يؤدي إلى فرط الحساسية، بالاضافة إلى ولى حرمان الطفل من الرضاعة الطبيعية التي تتضمن الدفه والتواصل الجسدي. (Tarbox \& Bermudez, $r \cdot \mid \mathrm{V}: \vee$ ) • النوم، أو الطفل المرهق، أوضغوط أخرى خارج جلسات الأكل مثل التعرض للبلطجة......

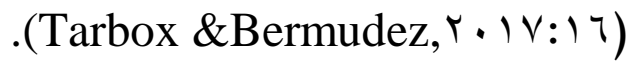




\section{العلاقة بين قصور المعالجة الحسية وانتقائية الطعام}

يعـانى العديد مـن الأطفال الأوتيزم مـن قصـور المعالجـة الحسية تحديدًا الحساسية الهفرطة والحساسية المنخفضة، وتؤثر هذه المشكلات على نموالطفل وقدرته على أداء أنشطة الحياة اليومية مثل الأكل، فهم لا يتعاملون مع المدخلات الحسية بنفس أسلوب أقرانهم العاديين. ويُعرف رولي، ومالوكس, وكوهانيك، وجلينون ( Roley,Mailloux, Kuhaneck \& 2007:2, قصسور المعالجـة الحسية بأنه عدم القدرة على معالجة المعلومـات المُستقبلة من خلال الحواس، ويكون القصور في الجهاز العصبي المركزي في الدماغ، وتكون نتيجة هذا القصور أن الطفل لا يستطيع الاستجابة للمعلومات الحسية والتصرف بطريقه متسقه ذات معنى، وكذلك يكون لديه صعوبة في استخدام المعلومات الحسية لتخطيط وتتظيم مـا

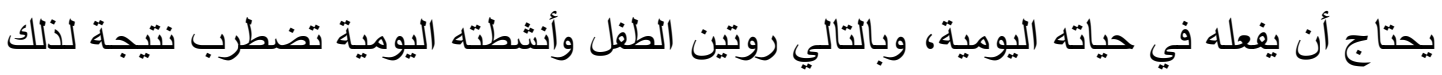

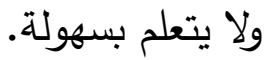

ولقد أشارت كاى (62-Key, 2001:61) إلى أن قصور المعالجة الحسية يؤثر على

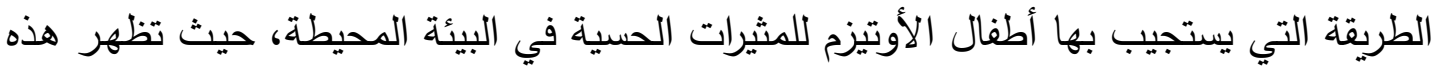

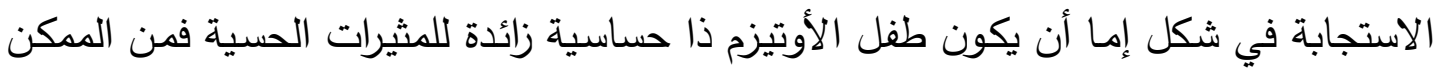

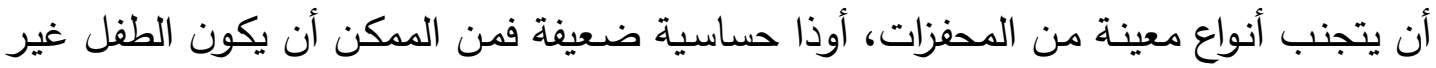

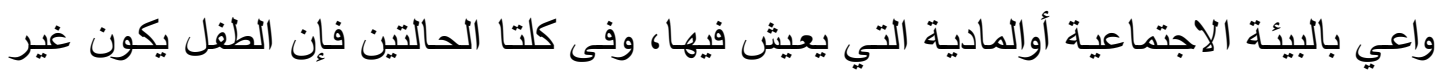
قادر على الاستجابة بنجاح للسياق البيئي، فيؤثر ذلك على الطريقة التي يتواصل بها وعلى التى قدرته على المشاركة في مواقف الحياة اليومية ويؤدي إلى انخفاض المشاركة الاجتماعية. كما أوضحت العديد من الدراسات إلى ارتفاع نسبة قصور المعالجة الحسية لدى الأوتيزم

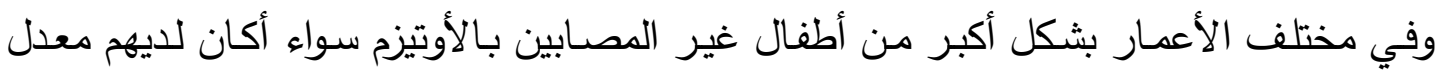

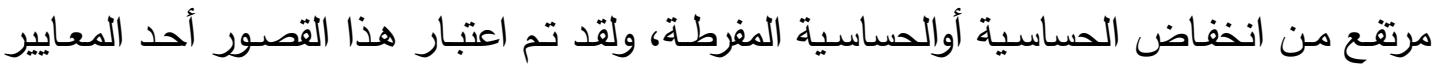
التشخصية للأوتيزم كما ورد في الدليل التصنيفي الخامس لرابطة الطب النفسي الأمريكية. وأوضتح ديمبسي وفورمان (Dempsey \&Foreman, 2001) أن التعديل الفعال

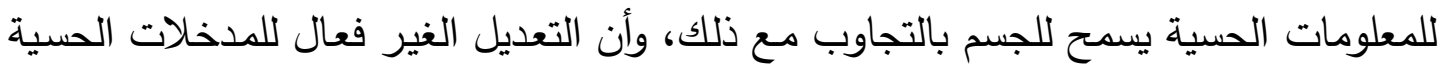

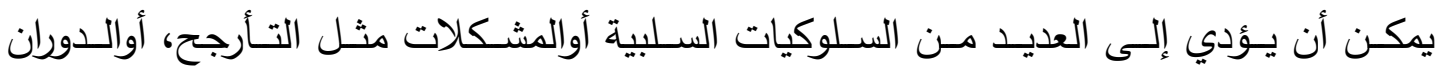

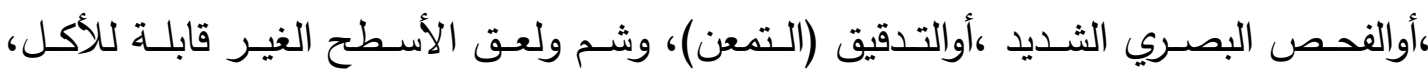

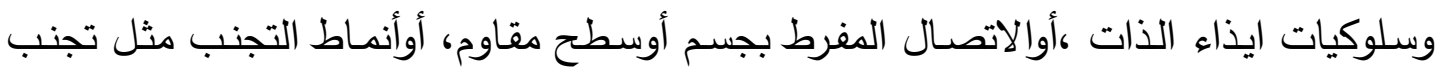


أطعدـة ذات ملمس معين، وتعرف جميع هذه السـلوكيات "بالسـلوكيات النمطيـة التكراريـة "،

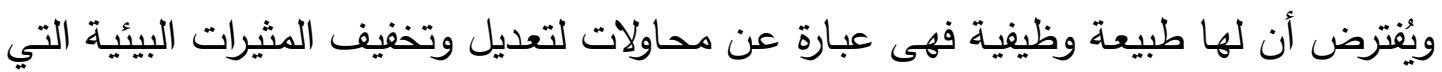

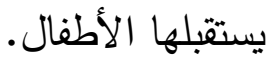

كما أثـار ميلر (Miller 2013:274) إلى أن قصور المعالجة الحسية يوثر على استعدادات الطفل والقدرة على المشاركة في تناول الوجبات، كما أن الحساسية اللمسية تؤثر

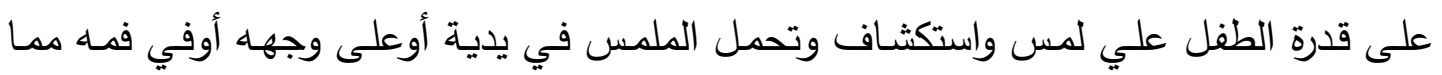

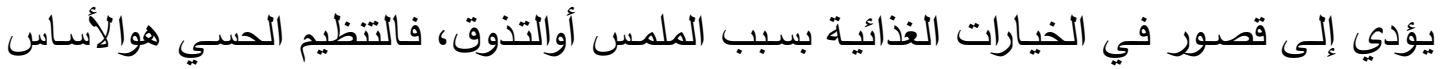

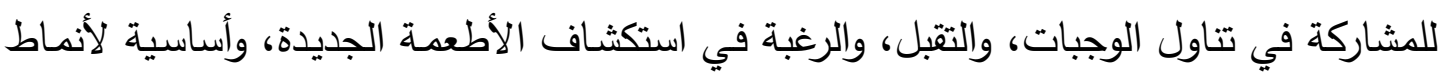

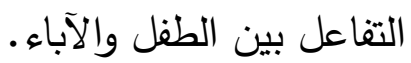

وأوضح سيرماك وكورتين وباندين (Cermak, Curtin \& Bandin, 2010:239) أن هناك عدة عوامل تسهم في انتقائية الطعام لدي الأوتيزم وأحد هذه العوامل هى الحساسية (لعانية

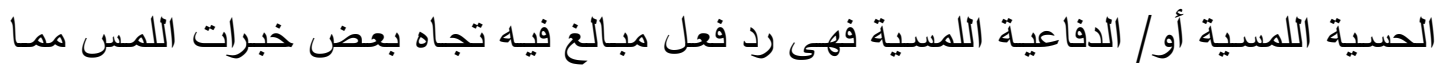
يؤدي غالبًا إلى استجابة سلوكية سلبية ملاحظة، فالأطفال الأوتيزم الذين يعانون من الدفاعية اللمسية غالبًا ما يرفضون المداعبة والعناق ويتجنبون اللمس، ومن المحتمل ان تسهم الحساسية اللمسية المبكرة في بعض قضايا تناول الطعام لدى الأوتيزم.

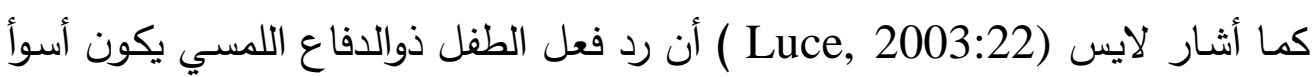

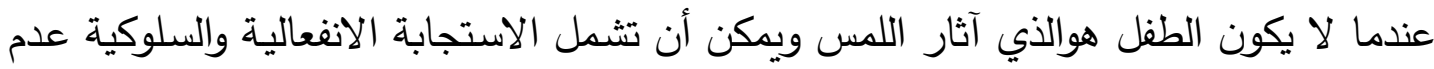

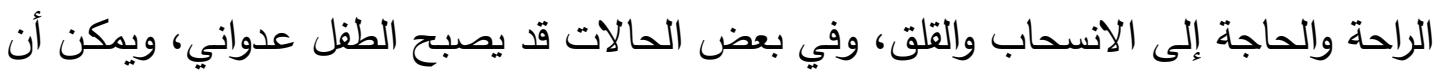

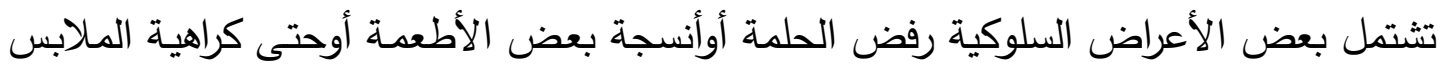

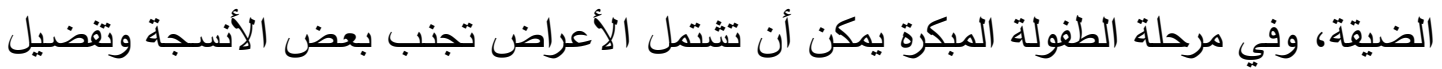
اللمس من الأثخاص المألوفين فقط والسلوك المؤذي للنفس وتجنب الاقتراب من الآخرين. وفي دراسة نادون، وفيلدمان، ودون، وجيزل Nadon, Feldman, , Dunn, \& (Nisel.,2011) (Nadon, الأوتيزم وجد أن الأطفال فى مجموعة الاختلاف المحدد (أي الذين حصلوا على درجة مرتفعة

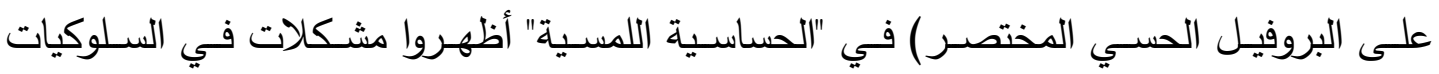
الاجتماعية في وقت الوجبات وكذلك تفضيل أنواع وألوان ودرجات حرارة غير معتادة للأطعمة. 
كمـا أوضــت النتائج أيضًا أن الأطفـال ذوي اضـراب طيف الأوتيـزم ومشكلات

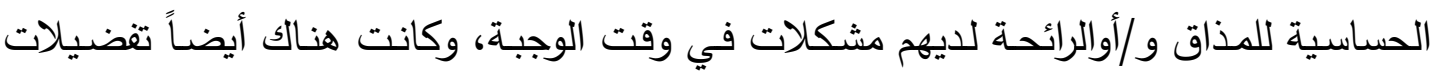
غير معتادة للأطعهـة، وكان لذلك أثر على استقلالية الأطفال أثناء الأكل بشكل أكبر من الناته الحساسية اللمسية، خاصة عند الأكل بدون مساعدة واستخدام أدوات المائدة مثل الشوكة. كما وجدت الدراسة ارتباط موجب دال أيضاً بين الحساسية البصرية والسمعية وبين عدد

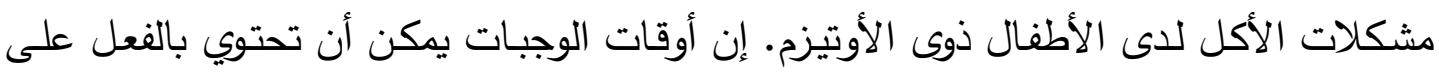

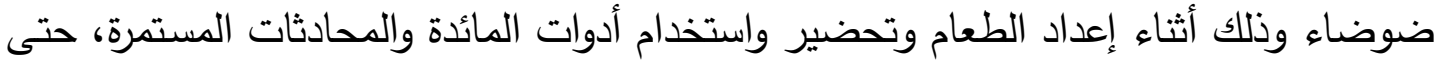

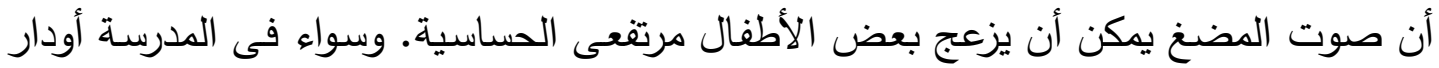
الرعاية فإن مستوى الضوضاء يكون أعلى من المعتاد فى منزل الطفل. كما كانت هناك كراهية

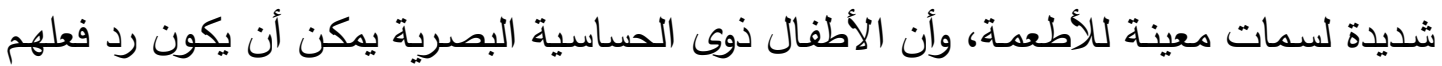

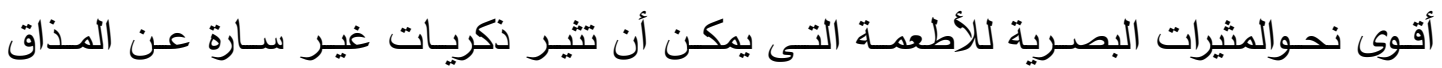

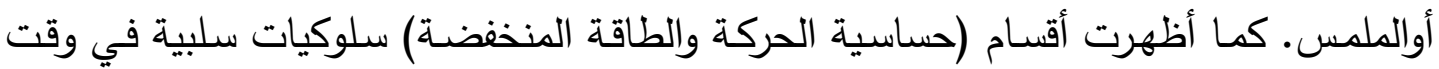
الوجبة، والعنصر الأقوى كان تفضيلات الطعام، مع وجود تأثير منخفض على استقلالية الأكل وتأثير أكبر على الحياة اليومية (يبدو وكأنه ضعيف العضلات). وفي دراسة لاشيزا (Lachiusa, 2013) أكدت على وجود علاقة موجبة ذات دلاتلاتية

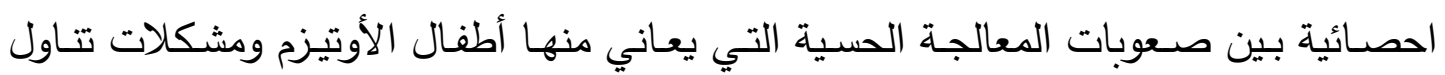

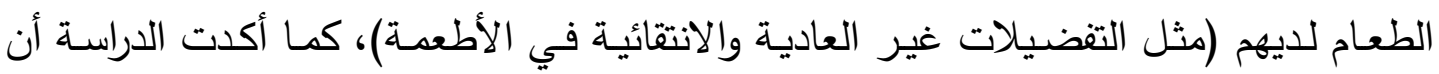
صعوبات تناول الطعام هى مشكلة شائعة لاى أطفال الأوتيزم تؤثر على صحتهم البدنية، آدائهم داخل الأسرة، وآدائهم في البيئات التعليمية وعلى روتينهم اليومي.

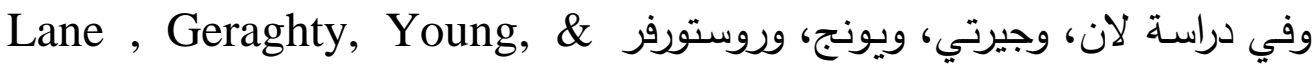
(Rostorfer.,2014)

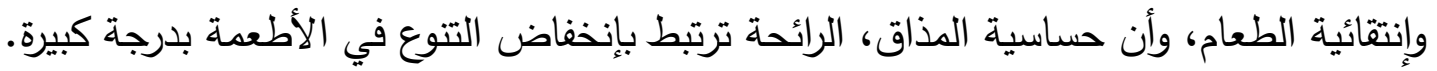

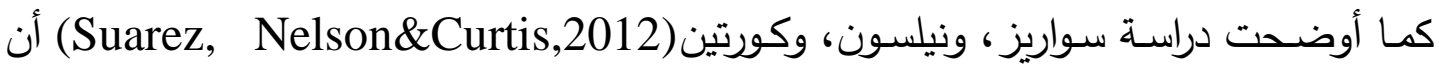

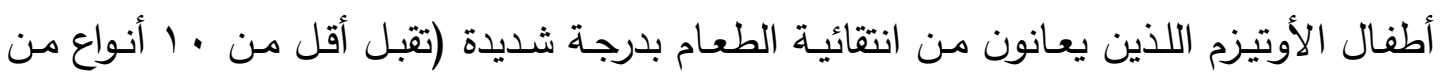
الأطعمة)، والذين يعانون من انتقائية الطعام بدرجة متوسطة(تقبل (1) - ـ بنوع من الأطعمة)

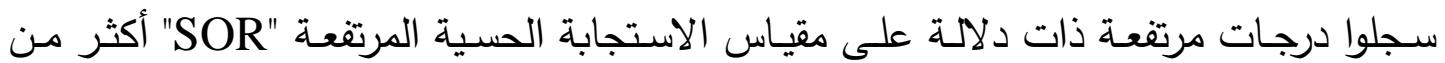
الأطفال اللذين يتقبلون (اب أوأكثر من الأطعمة) 
(Chistol, Bandini, كما أكد كيستول وبانديني ومست وفليبس وسيرماك وكورتين Must, Phillips, Cermak \& Curtin, 2018:589) أخصائي أمراض الكلام، وأخصـائي عـلاج وظيفي، وأخصـائي تغذيـة، وضـرورة تقديم أنشطة التكامل الحسي لخفض الحساسية الحسية، كمـا يجب أن تكون خطط العـلاج فرديـة ومراعاة الخصائص الحسية الفريدة لكل طفل أوتيزم. إجمالاً يتضح مما سبق أن مشكلة إنتقائية الطعام منتشرة بين الأطفال ذوى الأوتيزم، ولا يستطيع العديد من هؤلاء الأطفال معالجـة المعلومـات الآتيـة من المدخلات الحسية وتحديدًا الثمية والسمعية والبصرية واللمسية والتذوق بنفس أسلوب أقرانهم ذوى النموالطبيعى فى نفس العمر ، وأن هناك ارتباط قوي بين قصور المعالجة الحسية وإنتقائية الطعام. لذا تقترح الدراسات السابقة بضرورة وضع التدخلات الحسية في الإعتبار لعلاج إضطرابات الأكل وبخاصة انتقائية الطعام، ومن خـلال هذه التدخلات الحسية يستطيع الجهاز العصبي أن يعدل وينظم ويدمج المعلومات من البيئة مما يوفر أساس لاستجابات تكيفية مع البيئة.

\section{اجراءات اعداد بطاقة ملاحظة انتقائية الطعام لأطفال الأوتيزم}

تهدف هذه البطاقة إلى قياس مستوى انتقائية الطعام( قصور المعالجة الحسية أثناء الطعام- سلوكيات مصـاحبة أثناء تتاول الوجبات غير الدفضلة- رفض الأطعمة - قصور المهارات الحركية الفية) لدى أطفال أوتيزم الذين يتراوح أعمارهم من (r-7) سنوات.

\section{مبررات إعداد بطاقة ملاحظة انتقائية الطعاه لأطفال الأوتيزه :}

• لا توجد - في حدود علم الباحثة-أداة مقننة لفحص انتقائية الطعام لاى الأوتيزم.

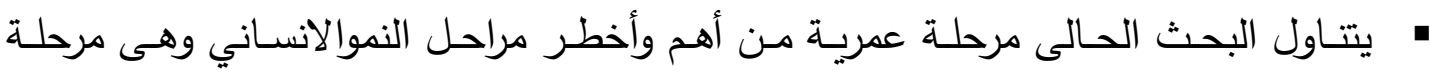

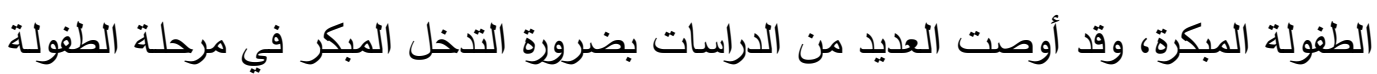
المبكرة مما يُقلل من حجم المشكلة وتأثيرها لدى الأوتيزم. 
وبنـاءً علـى مــا سـبق قامــت الباحثـة بإعـداد بطاقـة ملاحظــة انتقائيـة الطعـام لـدى

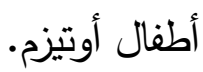

\section{خطوات إعداد بطاقة ملاحظة انتقائية الطعام لأطفال الأوتيزمر}

قامـت الباحثه بإعداد بطاقـة ملاحظة انتقائيـة الطعـام لأطفال الأوتيزم مـن خـلال

$$
\text { خطوتين وهى: }
$$

• إعداد الصورة الأولية لبطاقة ملاحظة انتقائية الطعام لأطفال الأوتيزم. • إعداد الصورة النهائية لبطاقة ملاحظة انتقائية الطعام لأطفال الأوتيزم.

\section{1- الصورة الأولبة لبطاقة ملإهظة انـنقائبة الطعام لأطلفال الأوتيزم}

\section{اتبعت الباحثه الخطوات الآتية:}

• مراجعة الإطار النظري، والدراسات السابقة الخاصة بمشكلات الأكل عند الأوتيزم وبخاصـة انتقائية الطعام، والدراسات التي تتاولت العلاقة بين قصور المعالجة الحسية وانتقائية الطعام لأطفال أوتيزم، وذلك كما ورد في الفصل الثاني والثالث من الدراسة. • الاطـلاع على بعض المقاييس الخاصـة بقصور المعالجة الحسية لدى الأوتيزم، وأدوات لقياس مشكلات تتاول الطعام لدى الأوتيزم، الأكل الحسي، والإفادة منها في بنـاء بطاقة الملاحظة، وتحديد أبعادها وتحديد التعريفات الإجرائية للأبعاد، وهذه الأدوات هى: (- معايير تشخيص اضطراب تتاول الطعام المقيد/ التجنبي (الإنتقائي) Avoidant/Restrictive Food Intake Disorder "ARFID" Brief Autism Mealtime - مقياس سلوكيات تتاول الوجبات المختصر لدى الأوتيزم (Lukens, 2005) " Inventory"Behavior r- القائمة الحسية لأطفال الأوتيزم"Larkey,2007)"The Sensory Checklist). ع - استبانة الوجبات في المنزل "Meals in Our Household questionnaire (MIOH)"

(Anderson, Must, Curtin \& Bandini, 2012) • قائمة الأكل الحسي "Sensory Eating Checklist ( Lachiusa,2013) "S 
ك- كقياس الاستجابة الحسية العالية "Suarez, "sensory over-responsivity (SOR)"

(Nelson\&Curtis, 2014)

V- قامت الباحثة باجراء دراسة استطلاعية في صورة سؤال مفتوح على عينة عشوائية من

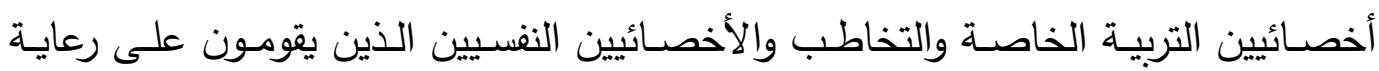
وتدريب أطفال الأوتيزم، وكذلك أمهات أطفال أوتيزم للتعرف على طبيعة مشكلة إنتقائية الطعام والمشكلات المصاحبة لها، وكانت صيغة السؤال المفتوح كالتالي: صف لي نظام طفلك/ طفلتك الذذائي في الوقت الحالي؟ • قامت الباحثة بعمل حصر للإجابات بناءً على نتيجة الدراسة الاستطلاعية.

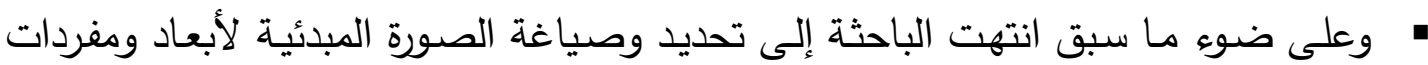

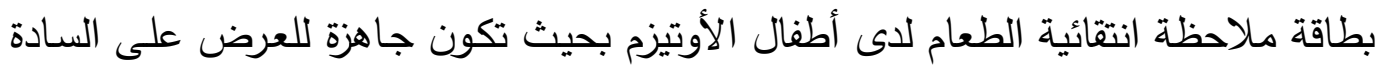
المحكمين وتتضمن الصورة المبدئية (• (1) مفردة موزعة على أربعة أبعاد كالآتي: • البعد الأول: قصور المعالجة الحسية (أثناء الطعام).

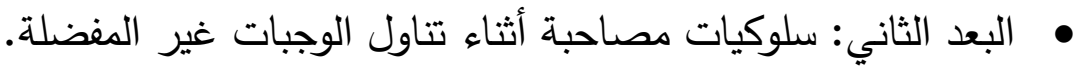

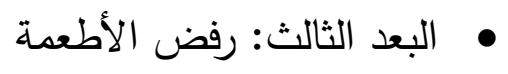

• البعد الرابع: قصور المهارات الحركية الفية.

\section{|r-1إعداد الصورة النهائية لبطاقة ملاهظة اننقائية الطعام الأطفال الأوتيزم:}

في ضوء ما سبق إنتهت الباحثة إلى صياغة الصورة المبدئية لبطاقة ملاحظة انتقائية الطعام لأطفال الأوتيزم. ثم قامت الباحثة بحساب الخصائص السيكومترية للبطاقة بعدة طرق:

\section{أولاً: حساب صدق بطاقة ملاحظتة انتقائية الطعام لأطفال الأوتيزم}

$$
\text { 1- الصـــل الظــاهــري }
$$

قامـت الباحثـة بعـرض بطاقـة الملاحظــة في صـورتها المبدئيسة على مجموعـة مـن المحكمين (• () من أساتذة الصحة النفسية وعلم النفس التربوي، وذلك للحكم على صلاحية لهية العبارات ومدى انتمائها وقدرتها على قياس موضوع بطاقة الملاحظة، ومدى سلامة الصياغة،

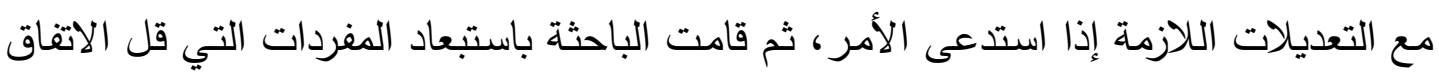
عليها بين المحكمين عن (•^\%) وتلخص الباحثه ما تم التوصل إليه في جدول (1). 


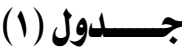

نسب إتفاق المحكمين لبطاقة ملاحظة انتقائية الطعام لأطفال الأوتيزه

\begin{tabular}{|c|c|c|c|c|c|c|c|}
\hline \multicolumn{2}{|c|}{ قصور المهارات الحركية الفمية } & \multicolumn{2}{|c|}{ روفـض الأطعمـــة } & \multicolumn{2}{|c|}{ تناول الوجبات غير المفضلة مصاتة أثناء } & \multicolumn{2}{|c|}{ قصور المعالجة الحسية } \\
\hline الاتفاق & مر & الاتفاق & مر & الاتفاق & مر & الاتفاق & مر \\
\hline$\% 9$ & 71 & $\% 1 \ldots$ & $\$ 1$ & $\% 9$. & rI & $\% 9$ & 1 \\
\hline$\% 9$. & Tr & $\% 9$. & $\xi r$ & $\% \wedge$. & rr & $\% 9$. & $r$ \\
\hline$\% \wedge$. & 9 & $\% \wedge$. & $\varepsilon r$ & $\% 1 \ldots$ & rr & $\% \wedge$. & $r$ \\
\hline $9 . \%$ & $7 \xi$ & $\% \wedge$. & $\llbracket \xi$ & $\% 9$. & $r \xi$ & $\% 9$. & $\xi$ \\
\hline$\% 1 .$. & 70 & $\%$. & \$o & $\% \wedge$. & ro & $\% \wedge$. & 0 \\
\hline$\% 9$. & 79 & $\% \wedge$. & $\leqslant 7$ & $\% \wedge$. & rq & $\%$. & 7 \\
\hline$\% 9$ & IV & $\% 9$ & \&r & $\% \wedge$. & rr & $\% \wedge$. & $r$ \\
\hline$\% \wedge$ & 71 & $\% 1 \ldots$ & $\{\Lambda$ & $\% 9$. & $r \Lambda$ & $\%$. & $\wedge$ \\
\hline$\% 9$. & 79 & $\% 1 \ldots$ & $\varepsilon 9$ & $\% 9$. & $r q$ & $\% \wedge$. & 9 \\
\hline$\% 1 \ldots$ & v. & $\% 1 \ldots$ & 0. & $\% \wedge$. & r. & $\% 1 \ldots$ & 1. \\
\hline$\% 9$. & n & $\% 1 \ldots$ & 01 & $\% 9$. & rl & $\% 1 \ldots$ & 11 \\
\hline$\% 9$. & rr & $\% 1 \ldots$ & or & $\% 1 \ldots$ & rr & $\% 1 \ldots$ & ir \\
\hline$\% \wedge$. & Vr & $\% 1 \ldots$ & or & $\% 9$. & r & $\% 1 \ldots$ & ir \\
\hline$\% 9$ & v\& & $\% 1 \ldots$ & $0\}$ & $\% 9$. & r६ & $\% 1 \ldots$ & $1 \varepsilon$ \\
\hline$\% 1 \ldots$ & vo & $\% 1 \ldots$ & $\Delta 0$ & $\% \wedge$. & ro & $\% 1 \ldots$ & 10 \\
\hline$\% 9$. & vi & $\% 1 \ldots$ & 07 & $\% 9$. & $m$ & $\% 1 \cdots$ & 17 \\
\hline$\% 9$. & rv & $\% 1 \ldots$ & or & $\% 1 \ldots$ & rr & $\% 1 \ldots$ & iv \\
\hline$\% \wedge$ & $\mathrm{VA}$ & $\% 1 \ldots$ & $\Delta \Lambda$ & $\% 9$. & $r \Lambda$ & $\% 1 \ldots$ & 11 \\
\hline$\% 9$. & vq & $\% 1 \ldots$ & 89 & $\% 9$. & rq & $\% \wedge$. & 19 \\
\hline$\% 9$. & A. & $\% 1 \ldots$ & 7. & $\%$. & $\xi$ & $\% \wedge$. & r. \\
\hline
\end{tabular}

ثم قامـت الباحثـة بـإجراء التعديلات في صـياغة بعض المفـردات بنـاءً على آراء

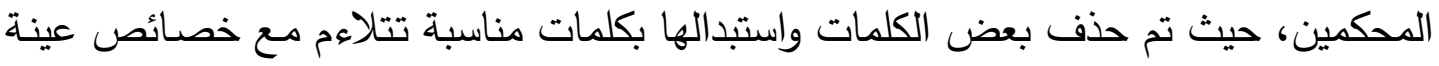


في ضوء ذلك قامت الباحثة باجراء التعديلات اللازمة للبطاقة والتي تكونت من (•^)

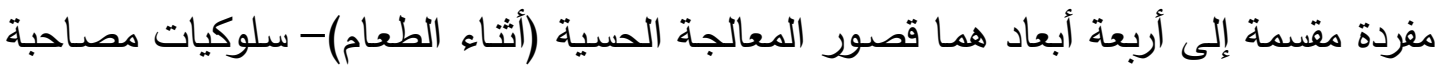

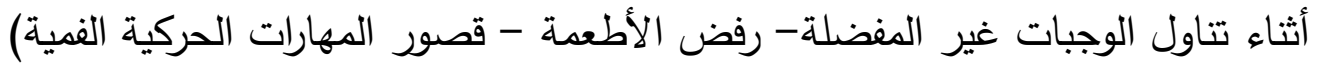
r - صدق المقارنة الطرفية لبطاقة ملاحظة انتقائية الطعام لأطفال الأوتيزم

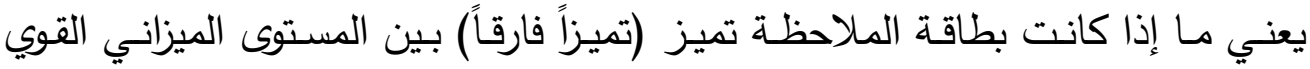

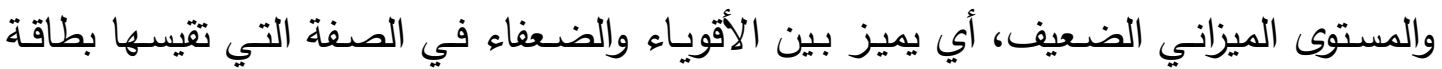

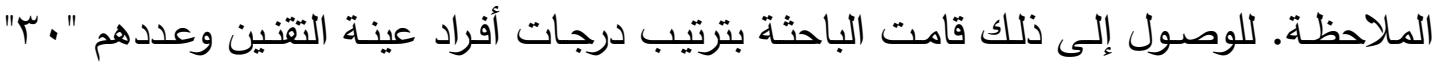
طفل على بطاقة الملاحظة ترتيبا تتازليا، ثم قام بعزل "Yr \% \% " من العدد الكلي للدرجات من

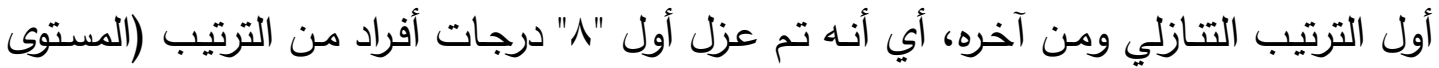

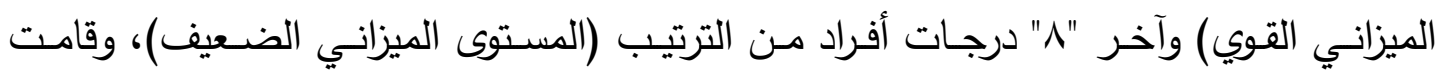

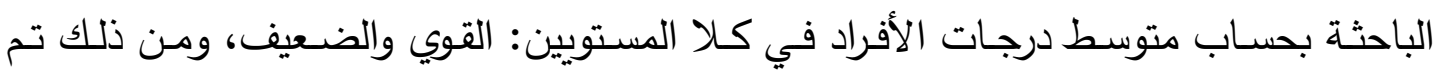
حساب الفرق القائم بين متوسط درجات الأفراد في المستوى الميزاني، وللتعرف على على مدى دلالية

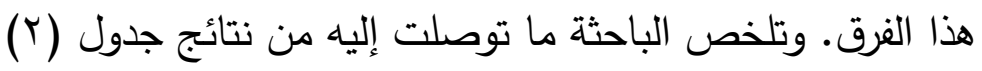

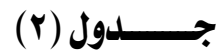

نتائج مان وتني لدراسة الفروق بين المجموعات الطرفية (الإرباعى الأعلى والإرباعى الأدنى)

لبطاقة ملاحظة انتقائية الطعام لأطفال الأوتيزه

\begin{tabular}{|c|c|c|c|c|c|c|c|}
\hline مستوى & قيمة Z & قيمة U U & مجموع & متوسط & ن & المجموعة & لأطفال الأوتيزه \\
\hline \multirow{2}{*}{,. } & \multirow{2}{*}{$r, \mathrm{YYA}-$} & \multirow{2}{*}{$\cdot, \cdots$} & $\ldots, \cdot$. & ir,o.e. & $\wedge$ & 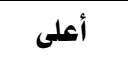 & \multirow{2}{*}{ الحسية (أثناء الطعام) } \\
\hline & & & $r q, \cdots$ & $\{, 0 .$. & $\wedge$ & أدنى & \\
\hline \multirow[b]{2}{*}{, } & \multirow[b]{2}{*}{ r, TVq- } & \multirow[b]{2}{*}{ •, $\cdots$} & $\cdots, \cdots$ & Ir,o... & $\wedge$ & أعلى & \multirow{2}{*}{ سلاوليات الوحاحباتة أثناء } \\
\hline & & & $r q, \cdots$ & $\{, 0 . \cdot$ & $\wedge$ & أدنى & \\
\hline \multirow{2}{*}{,. 1} & \multirow{2}{*}{ r, זAA- } & \multirow{2}{*}{$\cdot, \cdots$} & $1 \cdots, \cdot \cdot$ & $\mid r, 0 . \cdot$ & $\wedge$ & أعلى - أعلى & \multirow{2}{*}{ رفض الأطعمة } \\
\hline & & & $m, \cdots$ & $\varepsilon, 0 . \cdot$ & $\wedge$ & أدنى & \\
\hline \multirow{2}{*}{,. 1} & \multirow{2}{*}{$r, r T A-$} & \multirow{2}{*}{$\cdot, \cdots$} & $1 \cdots, \cdot$, & $\mid r, 0 .$. & $\wedge$ & أعلى & \multirow{2}{*}{ قصور المهارات الحركية } \\
\hline & & & $r q, \cdots$ & $\{, 0 \cdot \cdot$ & $\wedge$ & 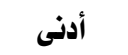 & \\
\hline \multirow{2}{*}{$\bullet, \cdot 1$} & \multirow{2}{*}{$r, r v 1-$} & \multirow{2}{*}{, , $\cdots$} & $1 \cdots, \cdot \cdot$ & ir,o... & $\wedge$ & & \multirow{2}{*}{ اللدرجة الكلية } \\
\hline & & & $r q, \cdots$ & $\varepsilon, 0 . \cdot$ & $\wedge$ & أدنى & \\
\hline
\end{tabular}




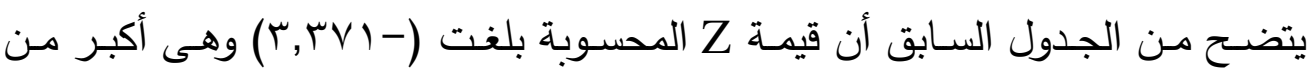

القيمة الحدية (דو, (1)، والمتوسط في المجموعتين الارباعي الأعلى والأرباعي الأدنى متفاوت

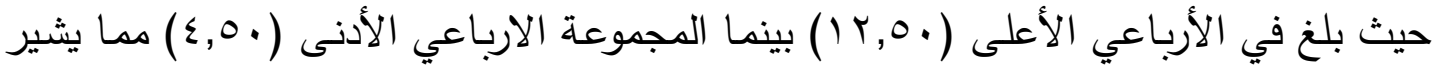
إلى وجود فروق دالة إحصائياً بين متوسطى رتب درجات أفراد المجموعتين الأرباعي الاعلى الاعى والارباعي الادنى لصـالح الارباعي الاعلى وهذا يؤكد قدرة بطاقة الملاحظة على التميز بين المرتفعين والمنخفضين مما يعني تمتع بطاقة الملاحظة وأبعادها بصدق تمييزي قوي.

ثانيًا: ثبات بطاقته ملاحظتة انتقائية الطعام لأطفال الأوتيزم: ا - طريقة إعادة تطبيق الاختبار:

وتمَّ ذلك بحساب ثبات بطاقة ملاحظة انتقائية الطعام لدى أطفال أوتيزم من خـلال

r • ( إعادة تطبيق الاختبار بفاصل زمنى قدره أسبوعين وذلك على عينة التقنين والتي بلغت طفل وطفلـة)، وتم اسـتخراج معـاملات الارتبـاط بين درجـات أطفـال العينـة باسـخدام معامـل بيرسـون (Pearson)، وكانـت جميـع معـاملات الارتبـاط لأبعـاد بطاقـة الملاحظــة دالـة عنـــ ( 1 ( • ) مما يشير إلى أنَّ بطاقة الملاحظة تعطى نفس النتائج تقرببًا إذا ما استخدم أكثر من مرَّة تحت ظروف مماثلة وبيان ذلك في الجدول الآتي:

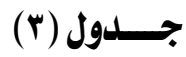

نتائج الثبات بطريقة إعادة الاختبار لبطاقة ملاحظة انتقائية الطعامل لأطفال الأوتيزم

\begin{tabular}{|c|c|c|}
\hline 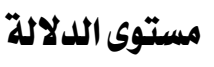 & معامل الارتباطبين التطبيقين الأول والثانى & انتقائية الطعاه لأطفال الأوتيزم \\
\hline$\cdot,+1$ & $* * \bullet, 9$ qr & قصـــور المعالجــة الحسيـة (أثناء الطعام). \\
\hline$\cdot, \cdot 1$ & $* *, \mathrm{YIA}$ & المفضلة \\
\hline \multirow[t]{2}{*}{$\cdot, \cdot 1$} & $* * \bullet, 77 r$ & رفــض الأطعمـــة \\
\hline & $* * \bullet, \vee 00$ & قصور المهارات الحركية الفمية \\
\hline$\cdot, \cdot 1$ & $* * *, 9 \cdot r$ & الــلدرجــــة الكليـــة \\
\hline
\end{tabular}

يتضح من خلال الجدول السابق وجود علاقة ارتباطيه دالة إحصائيًا بين التطبيق الأول

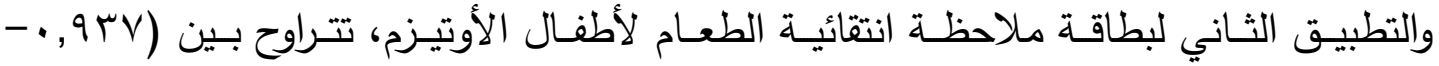

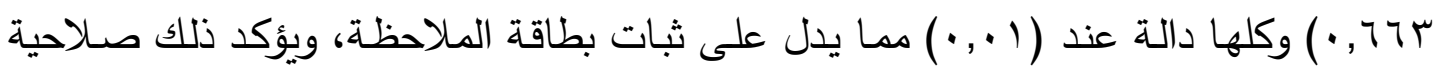
بطاقة ملاحظة انتقائية الطعام لأطفال الأوتيزم لقياس السمة التي وُضع من أجلها. 


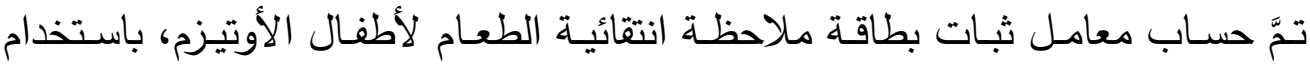

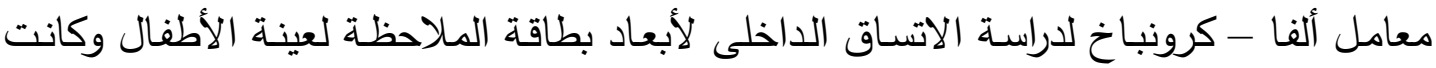

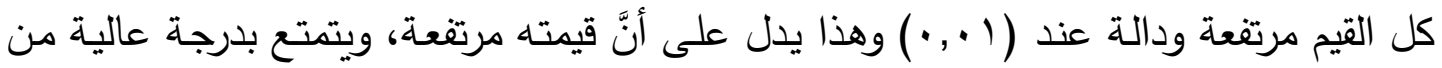
الثبات، وبيان ذلك في الجدول الآتي:

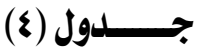

معاملات ثبات بطاقة ملاحظة انتقائية الطعام لأطفال الأوتيزر باستخذام معامل ألفا- كرونباخ

\begin{tabular}{|c|c|c|c|}
\hline مستوى الثبات & أ ألفا-كرونباخ & أبعاد بطاقة ملاحظة انتقائية الطعام لأطفال الأوتيزمر & هـ \\
\hline مرتفعة & •, 9 • & قصور المعالجة الحسية (أثناء الطعام) & 1 \\
\hline مرتفعة & - 949 & سلوكيات مصاحبة أثناء تناول الوجبات غير المفضلة & $r$ \\
\hline مرتفعة & •,9TY & رفــض الأطعمــة & $r$ \\
\hline 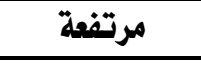 & •,9TY & قصور الههارات الحركية الفمية & $\xi$ \\
\hline مرتفعة & $\cdot, 911$ & السـلدرجسة الكليــة & \\
\hline
\end{tabular}

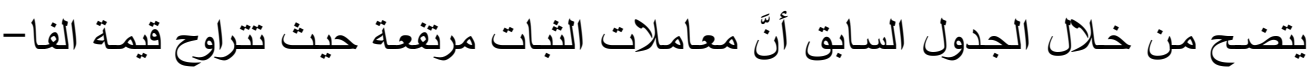

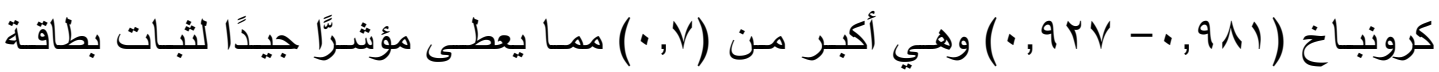

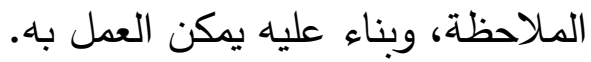

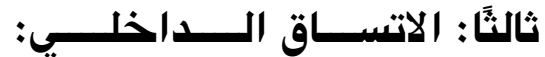
1 - مصفوفة الارتباط لبطاقة ملاحظة الاتبات التائية الطعام لأطفال الأوتيزم: تم حساب معاملات الارتباط باستخدام مُعامل بيرسون (Pearson) بين أبعاد بطاقة لإنة

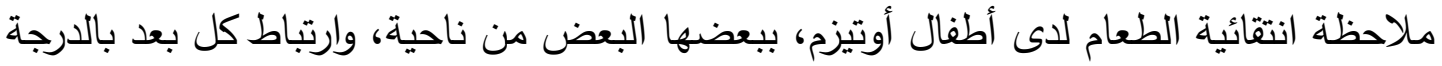

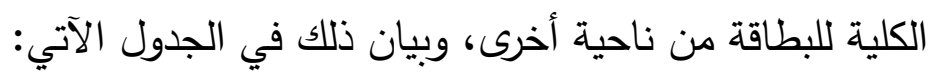

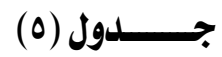

مصفوفة ارتباطات بطاقة ملاحظة انتقائية الطعام لأطفال الأوتيزه

\begin{tabular}{|c|c|c|c|c|c|}
\hline 0 & $\xi$ & r & $r$ & 1 & انتقائية الطعام لأطفال الأوتيزه \\
\hline & & & & - & ق قصور المعالجة الحسية(أثناء الطعام). \\
\hline & & & - & $* * *, 904$ & سلوكيات مصاحبة أثناء تناول الوجبات غير المفضلة \\
\hline & & - & $* * *, A Y Y$ & $* * \bullet, \wedge \leqslant 0$ & رفــض الأطعمــة \\
\hline & - & $* * *$, q Y & $* * *$, A૬т & $* * *$, v99 & قصور المهارات الحركية الفمية \\
\hline - & **•, , १६r & ***, $97 \mathrm{r}$ & $* *, 970$ & $* * *, 9 \leqslant \varepsilon$ & السـلدرجــة الكليــة \\
\hline
\end{tabular}

** * دال عند مستوى دلالة (1., (•) 
يتضح من جدول (0) أنَّ جميع معاملات الارتباط تتراوح ما بين (070, • - V99, • ) وهي

دالة عند مستوى دلالة (1 . . •) مما يدل على تمتع بطاقة الملاحظة بالاتساق الداخلي والثبات.

r - الاتساق الداخلي (المفردات مع الارجة الكلية) لبطاقة ملاحظة انتقائية الطعام لأطفال الأوتيزم: وذلكلك مـن خـالل درجـات عينـة التقنـين (الاســطلاعية) بإيجـاد معامـل ارتبـاط بيرسـون

بين درجات كل مفردة والدرجة الكلية لبطاقة الملاحظة، وبيان ذلك في الجدول الآتي: (Pearson)

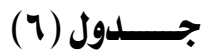

\section{معاملات الارتباط بين درجات كل مفردة والدرجة الكلية}

\section{لبطاقة ملاحظة انتقائية الطعام للدى أطفال الأوتيزمه =•r}

\begin{tabular}{|c|c|c|c|c|c|c|c|}
\hline \multicolumn{2}{|c|}{ قصور المهارات الحركية الفمية } & \multicolumn{2}{|c|}{ 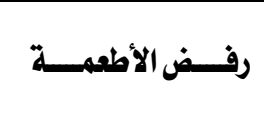 } & \multicolumn{2}{|c|}{ سلوكيات مصاحبة أثناي تناول الوجبات غير المفضلة } & \multicolumn{2}{|c|}{ قصور المعالجة الحسية } \\
\hline الارتباط & مر & الارتباط & مر & الارتباط & مر & الارتباط & مر \\
\hline$* * \bullet, \vee \wedge 1$ & 71 & $* * \cdot, 7 \cdot 0$ & $\$ 1$ & $* \bullet, \leqslant \leftleftarrows \wedge$ & rl & $* * \bullet, \wedge \varepsilon \bullet$ & 1 \\
\hline$* * \bullet, \Lambda \leqslant \Lambda$ & $9 r$ & $* * \bullet, 077$ & $\xi r$ & $* * \bullet, \wedge \vee \uparrow$ & rr & $* * \bullet, A \vee Y$ & $r$ \\
\hline$* * \bullet$, oro & $9 r$ & $* * \bullet,\{9 々$ & $\varepsilon r$ & $* * \bullet$, Aro & rr & $* \bullet, \llbracket \leftleftarrows \wedge$ & $r$ \\
\hline$* * \bullet, \wedge \vee q$ & $9 \varepsilon$ & **•, ovo & $\llbracket \xi$ & $* * \bullet, \wedge \leqslant \wedge$ & rz & $* *, \wedge \mid Y$ & $\varepsilon$ \\
\hline$* *, \sum V r$ & 70 & ***,orr & §o & $* * \bullet,\{\wedge \wedge$ & ro & $* * \bullet, \wedge \vee \neg$ & 0 \\
\hline **•, Trr & 79 & ***, 7 Y & $\Sigma \eta$ & $* * \bullet, \wedge \leqslant \wedge$ & rq & $* * \bullet,\{\wedge \wedge$ & 7 \\
\hline$* *$, VYY & IV & $* *, 7 \mid r$ & $\{r$ & $* *, \wedge \varepsilon \bullet$ & $r V$ & $* \bullet,\{\Sigma Y$ & $\checkmark$ \\
\hline$* *$, ov. & 71 & $* * \bullet, 099$ & $\xi \wedge$ & $* *, \wedge \mid \vee$ & $r \wedge$ & $* *, \wedge \| 11$ & $\wedge$ \\
\hline$* *, 7 r r$ & 79 & $* * \bullet, 0 \leqslant V$ & $\leqslant 9$ & $* * \bullet$, Yor & $r q$ & $* *, \vee \vee \wedge I$ & 9 \\
\hline ***, Trr & v. & $\cdot, \Delta \gamma * * *$ & 0. & ***,Arr & $r \cdot$ & $* * \bullet, 70 \Lambda$ & 1. \\
\hline$* * *$, รq. & n & ***, or. & 01 & $* * \bullet, A Y Y$ & ri & $* * \bullet, \wedge \vee \neg$ & 11 \\
\hline$* * \bullet, 01$ & vr & $* * *, \wedge \varepsilon$ & or & $* * \cdot,\{\wedge \uparrow$ & rr & $* \bullet,\{\leqslant \wedge$ & ir \\
\hline$* *$, IV० & vr & $* * *, \wedge \vee Y$ & or & $* * \bullet, \wedge \xi \bullet$ & $r r$ & $* * \bullet, \Lambda \leqslant \Psi$ & ir \\
\hline$* * \bullet,\{\wedge \uparrow$ & $v \varepsilon$ & $* \bullet,\{\leqslant \wedge$ & $\Delta \xi$ & ***,ATr & rร & $* * \bullet, \wedge \vee \neg$ & is \\
\hline$* *, 7 \cdot \xi$ & vo & $* * \bullet, \wedge \leqslant r$ & 00 & $* *$, AYr & ro & $* *,\{\wedge \wedge$ & 10 \\
\hline ***, ova & vq & $* * \bullet, 094$ & 07 & $* \bullet,\{r V$ & m & $* *, \llbracket \llbracket \Psi$ & 17 \\
\hline$* * \bullet, 00 \xi$ & vr & $* * *$, AVY & or & $* *, 01$ & $r V$ & $* * \bullet, \wedge \leqslant \wedge$ & iv \\
\hline$* *,, 7 \mid r$ & rA & $* *,\{\wedge \wedge$ & $\Delta \wedge$ & $* *, 7 I r$ & $r \Lambda$ & $* *$, Avч & in \\
\hline ,, Y & va & $* *$, * $\{\leqslant \Psi$ & 89 & $* * \cdot, 7 \cdot \xi$ & $r q$ & $* *,, \llbracket \leqslant \wedge$ & 19 \\
\hline$\cdot, \cdot r r \cdot$ & $\Lambda \cdot$ & $* * \bullet, \wedge 11$ & 7. & ***, & $\xi$ & $* * \bullet, \wedge \vee \nearrow$ & r. \\
\hline
\end{tabular}

يتضـح من جدول (7) أنَّ جميع مفردات بطاقـة ملاحظـة انتقائيـة الطعام لدي أطفال

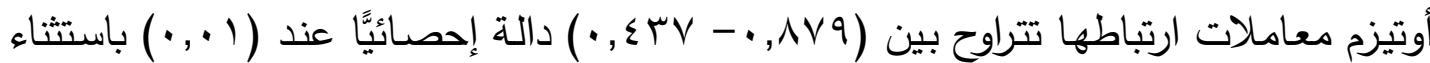

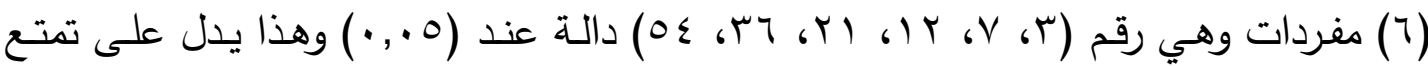


بطاقة الملاحظة بدرجة صدق مرتفعة، كما تم استبعاد (ץ)عبارتان، إذ تبين أنَّ معاملات ارتباط هذه الدفردات بالبطاقة معاملات صغيرة القيمة، وغير دالة إحصائيَّا مما يعنى أنَّ هذه المفردات آنسات غير صادقة، مما يستدعى استبعادها تمامًا من بطاقة الملاحظة.

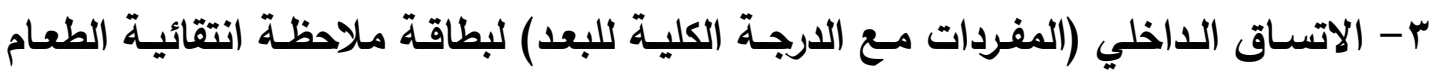
لأطفال الأوتيزم وقامت الباحثة بإيجاد التجانس الداخلي لبطاقة الملاحظة عن طريق حساب معاملات

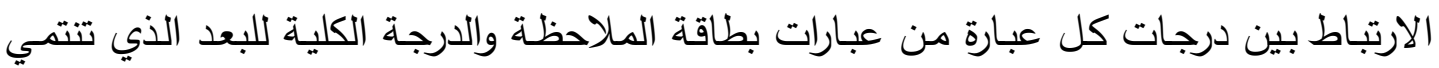
إليه، وبيان ذلك في الجدول الآتي:

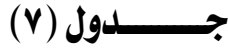

معاملات الارتباط بين درجات كل مفردة واللدرجة الكلية للبعد الذني تنتعي له

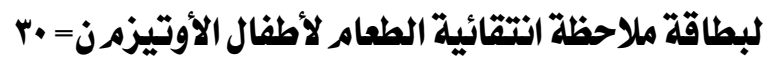

\begin{tabular}{|c|c|c|c|c|c|c|c|}
\hline \multicolumn{2}{|c|}{ قصور المهارات الحركية الفمية } & \multicolumn{2}{|c|}{ 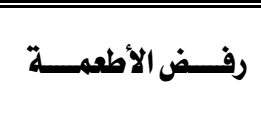 } & \multicolumn{2}{|c|}{ سلوكيات مصاحبة أثناي تناول } & \multicolumn{2}{|c|}{ قصور المعالجة الحسية } \\
\hline الارتباط & هـ & الارتباط & مـ & الارتباط & مر & الارتباط & مر \\
\hline$* *, 794$ & 71 & $* * \bullet, Y 0\}$ & $\$$ & $* \bullet, \xi * \wedge$ & $r$ & $* * \bullet, 9 \cdot 0$ & 1 \\
\hline$* * \cdot, 770$ & 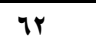 & $* *, V I \Lambda$ & $\{r$ & $* * \bullet, \wedge 91$ & rr & $* *$, aro & $r$ \\
\hline$* * *, 794$ & 94 & $* * \bullet, 07 \xi$ & $\{r$ & $* *, \wedge$ ฯ & rr & $* * \bullet, \sum \wedge r$ & $r$ \\
\hline ***,VIr & $7 \xi$ & $* * \bullet$, OIV & $\xi \xi$ & $* * \bullet, \wedge \wedge r$ & 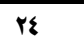 & $* * \bullet, \wedge A r$ & $\xi$ \\
\hline$* \bullet, r \wedge 9$ & 70 & $* * \bullet,\{19$ & \$o & $* * \bullet,\{0 \Lambda$ & ro & $* *$, 9ro & 0 \\
\hline$* * \bullet, \wedge 19$ & 79 & $* *, 7 \wedge 1$ & $\{1$ & $* * \bullet, \wedge 9 \xi$ & rq & $* \bullet,\{r q$ & 9 \\
\hline ***, Trr & Tr & $* *, 7$ TY & $\xi V$ & $* * \bullet, 9+1$ & rV & $* \bullet, \sum \Sigma Y$ & $v$ \\
\hline$* * *, 7 \cdot 0$ & 71 & $* * \cdot, 770$ & $\xi \Lambda$ & $* * *, \wedge \wedge r$ & $r \Lambda$ & $* * \bullet, \wedge 9$ & $\Lambda$ \\
\hline$* * \bullet, \wedge 19$ & 79 & $* * \cdot, V \cdot r$ & $\$ 9$ & $* *, \wedge \mid \gamma$ & $r q$ & $* * \bullet, \wedge Y r$ & 9 \\
\hline$* * \bullet, \wedge 19$ & v. & $* * *, Y Y I$ & 0. & $* * \bullet, q \cdot r$ & $r$. & $* *, V I r$ & 1. \\
\hline$* \bullet,\{r r$ & $n$ & $* *$ **, YYI & 01 & $* *, \wedge \vee q$ & $H$ & ***,aro & 11 \\
\hline$* * \bullet, 00$ & VY & $* * \bullet, Y \leqslant 0$ & or & $* * *,\{\wedge \uparrow$ & $r r$ & $* * \bullet,\{\wedge r$ & $\overline{i r}$ \\
\hline$* * \bullet, 01\}$ & vr & $* * \bullet, \vee Y \xi$ & or & $* * \bullet, 9 \cdot 1$ & rr & $* * \bullet, q \cdot V$ & ir \\
\hline$* * \bullet, 770$ & v\& & $* \bullet, \leqslant 7$ & $0 \xi$ & $* * \bullet, q \cdot r$ & rq & $* * \bullet, 9$ Yro & $1 \xi$ \\
\hline$* *$, OYA & ro & $* *, Y \Sigma Y$ & 00 & $* * \bullet, \wedge \vee q$ & ro & $* * \bullet,\{r q$ & 10 \\
\hline$* * \bullet, 00 r$ & vi & $* *, 7$ ro & 07 & *•,हrr & $r q$ & $* * \bullet$, $\{\xi Y$ & 17 \\
\hline$* * \bullet, 091$ & $r v$ & **•, YVY & or & $* * \bullet, 009$ & $r v$ & ***, 9ro & iv \\
\hline$* * \bullet, \vee q$. & VA & $* \bullet,\{r \Lambda$ & $\Delta \Lambda$ & $* * \bullet,\{91$ & $r \Lambda$ & $* * \bullet, 9$ Yro & 11 \\
\hline$\cdot, \cdot Y r \cdot$ & $\mathrm{vq}$ & $* \bullet, \xi r_{\bullet}$ & 89 & $* *, 717$ & $r q$ & $* * \bullet,\{\wedge \uparrow$ & 19 \\
\hline$\cdot, \cdot r q$. & A. & $* *, \mathrm{VIA}$ & 7. & $* * \bullet,\{91$ & $\xi$ & $* * \bullet, 9$, & $r$. \\
\hline
\end{tabular}


يتضح من الجدول السابق أنَّ جميع مفردات بطاقة ملاحظة انتقائية الطعام لدى أطفال

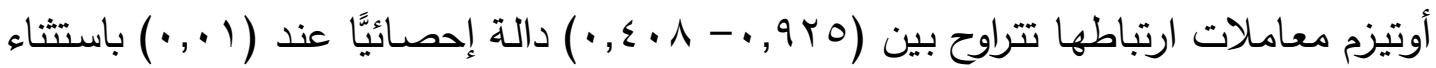

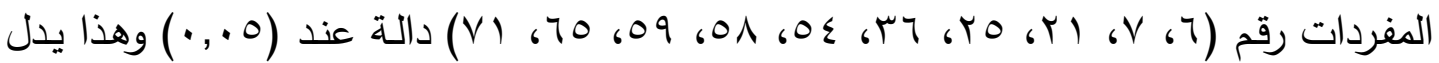
على تمتع بطاقة الملاحظة بدرجة صدق مرتفعة، كما تم استبعاد عبارتين.

\section{الصورة النهائية لبطاقة ملاحظة انتقائية الطعاه لأطفال الأوتيزم :}

\section{وصــف البطـــــــة}

تكون البطاقـة من (VA) مفردة في صـورتها النهائيـة موزعـة على أربعـة أبعـاد الاول قصور المعالجـة الحسية (أثتاء الطعام) وشمل (· ب) مفرده ،البعد الثاني سلوكيات مصـاحبة أثناء تتـاول الوجبات غير المفضلة ويشمل ( • ( ) مفردة، البعد الثالث رفض الأطعمـة ويشمل

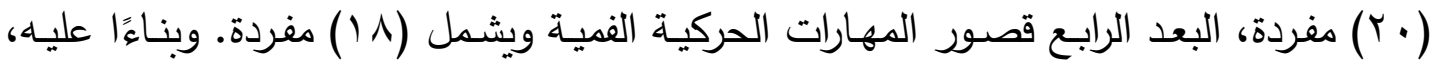
قامت الباحثة بإعداد الصورة النهائية لبطاقة ملاحظة انتقائية الطعام لأطفال الأوتيزم، وذلك بإعادة ترتيب مفردات بطاقة الملاحظة بصورة تبادلية بالنسبة للأبعاد الاربعة للبطاقة، بحيث لا تكون هناك مفردتان متتاليتان تقيسـان نفس البعد ،وتتم الاجابـة عن كل مفردة من مفردات البطاقة من خلال ثلاث بدائل هي: دائماً، أحيانًا، أبدًا، وتعطي درجات ب، Y ، ا علي الترتيب،

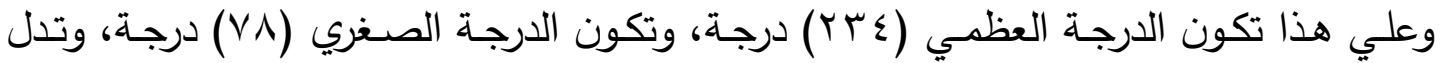
الدرجة العلياعلى مستوي مرتفع في حدة أعراض مشكلة انتقائية الطعام لدي أطفال أوتيزم، أمـا الدرجة المنخفضة فتلل علي انخفاضها، وتتضمن البطاقة الأبعاد التالية:

\section{البعد الأول: قصور المعالجة الحسيتة (أثناء الطعام)}

وهـو: عدم القدرة علي معالجـة المعلومـات المُستقبلة مـن خـلال الحواس أثناء تنـاول

الطعام، ويكون القصور في الجهاز العصبي المركزي في الدماغ، ويكون نتيجة هذا القصور أن الطفل لا يستطيع الاستجابة للمعلومات حسية والتصرف بطريقة متسقة ذات معني.

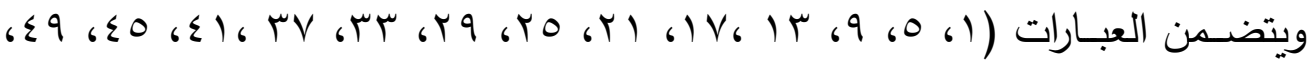

$$
\text { ( V7 6VT 679.7067) 6OV 60 }
$$




\section{البعد الثاني: سلوكيات مصاحبة أثناء تناول الوجبات غير المفضلة.}

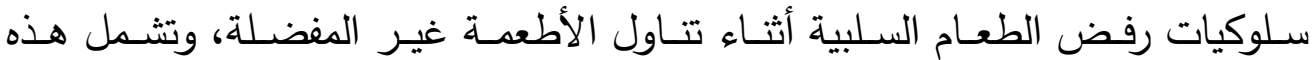
السلوكيات البكاء، نوبات الغضب، إيذاء الذات، قذف الطعام من على طاولة الطعام، وهذه السلوكيات تحدث استجابة لتقديم أطعمة غير مُفضلة.

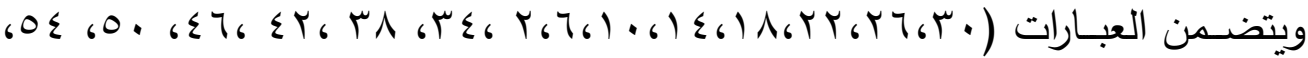

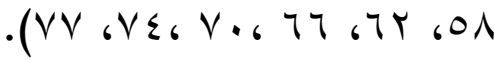

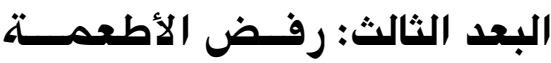

وهو: الأطعمة التي لا يتناولها الطفل بناءًا على استمارة التقييم الوظيفي.

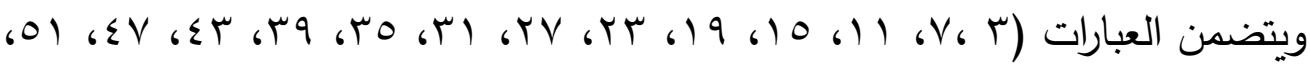

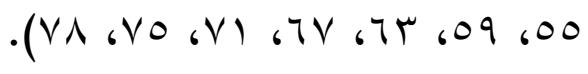

\section{البعد الرابع: قصور المهارات الحركيتة الفميتة.}

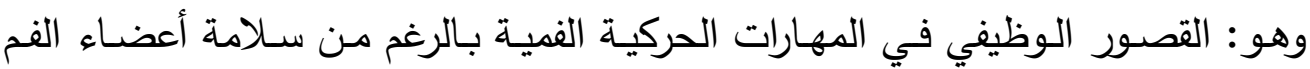

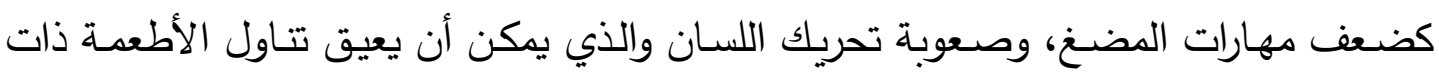

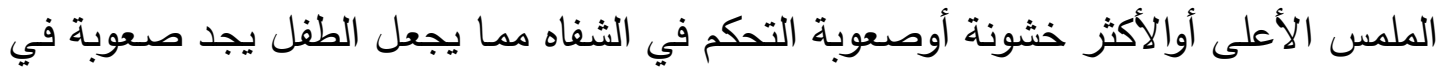
تناول الأطعمة المشتملة على السوائل.

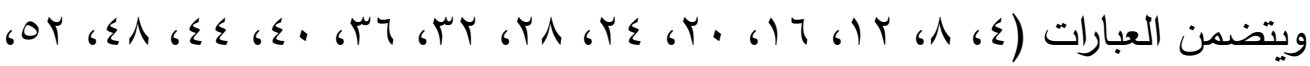

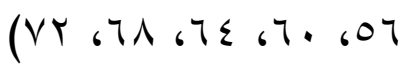

ومما سبق يتضح أنه يمكن تقييم وتشخيص انتقائية الطعام لدي أطفال الأوتيزم من خلال بطاقة ملاحظة انتقائية الطعام التي قامت باعدادها الباحثة حيث تمتعت بدرجة عالية من الصدق والثبات والاتساق الداخلي.

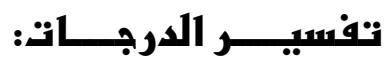
1- إذا حصل الطفل على درجة تتحصر بين VA: • با ا، يعني أن الطفل يعاني من الانتقائية بدرجة بسيطة.

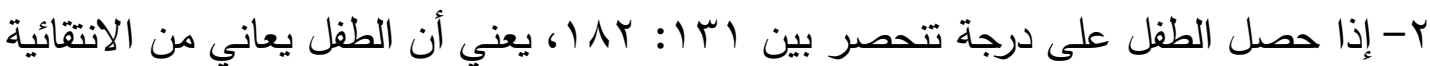
بدرجة متوسطة.

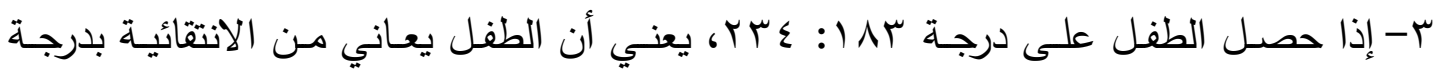
شديدة. 


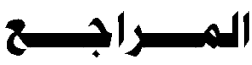

1- هشام عبد الرحمن الخولي (ع ( • Y). الاضطرابات النمائية (الأوتيزم -سأسبرجر كريت) بنها:

دار المصطفي للطباعة.

2- American Psychiatric Association. (2013). Diagnostic and statistical manual of mental disorders DSM-5 ,Fifth Edition, Washington DC: American Psychiatric Association.

3- Cermak, S,A., Curtin, C.,Bandini, L.(2010). Food selectivity and sensory sensitivity in children with autism spectrum disorders, J Am Dietetic Assoc,110(2):238-246.

4- Dempsey, I.\& Foreman, P. (2001). A review o f educational approaches forindividuals with autism. International Journal o $\mathrm{f}$ Disability, Development andEducation, 48 (1), 103-116.

5- Key, F. (2001). The relationship between sensory processing and self care for children with autism ages two to four, Retrrieved from Proquest Digital Dissertations(AAT 3027819).

6- Lachiusa,J.Z.(2013).Sensory Processing and the Self Care Task of Eating in Children with Autism, Retrrieved from Proquest Digital Dissertations (AAT 3589231).

7- Larkey,S.(2007). Practical Sensory Programmes For Students with Autism Spectrum Disorders, London: Jessica Kingsley Publishers.

8- Lane,A.E ， Geraghty,M.E, Young,G.S,\& Rostorfer,J.L.(2014). Problem Eating Behaviors in Autism Spectrum Disorder Are Associated With Suboptimal Daily Nutrient Intake and Taste/Smell Sensitivity, Infant, Child, \& Adolescent Nutrition, 6, (3), 172-180.

9- Ledford, J. R., \& Gast, D. L. (2006). Feeding problems in children with autism spectrum disorders: A review. Focus on Autism and Other Developmental Disabilities, 21, 156-166.

10- Levin, L., \& Carr, E. G. (2001). Food selectivity and problem behavior in children with developmental disabilities: Analysis and intervention,Behavioural Modification, 25, 443-470.

11- Luce, J. B. (2003).The effects of sensory motor therapy on the stereotypic movements of children with disorders on the autistic spectrum , $\{\mathrm{PhD}$. dissertation $\}$. Retrrieved from Proquest Digital Dissertations(AAT 308667). 
12- Lukens, C. T. (2005). Development and validation of an inventory to assess eating and mealtime behavior problems in children with autism, Retrrieved from Proquest Digital Dissertations (AAT 3192869).

13- Martins,Y, Young,R.L,\& Robson,D.C (2008) Feeding and Eating Behaviors in Children with Autism and Typically Developing Children, Journal of Autism and Developmental Disorders, 38,18781887.

14- Miller,j(2013). Recognizing and Addressing the Role of Food as a Sensory Regulation Strategy in Children With Autism, Infant, Child, \& Adolescent Nutrition, 5,5,274:282.

15- Milnes,S.M.(2011). The evaluation of a parent-implemented behavioral intervention for the treatment of food selectivity and mealtime behavior problems in children with autism spectrum disorders ,Retrrieved from Proquest Digital Dissertations (AAT 3488293).

16- Nadon, G., Feldman, D., Dunn, W.,\& Gisel, E., (2011). Association of sensory processing and eating problems in children with autism spectrum disorders. Autism Research and Treatment,1-8.

17- Patel, M. R., Piazza, C. C., Layer, S. A., Coleman, R., \& Swartzwelder, D. M. (2005). A systematic evaluation of food textures to decrease packing and increase oral intake in children with pediatric feeding disorders. Journal of Applied Behavior Analysis, 38(1), 89-100.

18- Provost,B., Crowe,T,K , Osbourn,P,L, McClain,C. , Skipper,B,J. (2010).Mealtime Behaviors of Preschool Children: Comparison of Children with Autism Spectrum Disorder and Children with Typical Development,Physical \& Occupational Therapy in Pediatrics, 30(3), 220-233.

19- Roley, S., Mailloux, Z., Kuhaneck, M. H., \& Glennon,T. (2007).Understanding Ayres sensory integration. AOTA Continuing Education, OT PRACTICE, 12,(17),1-8.

20- Chistol, L.T., Bandini, L.G., Must, A, Phillips, S., Cermak, S.A.,\& Curtin, C.(2018). Sensory Sensitivity and Food Selectivity in Children with Autism Spectrum Disorder,J Autism Dev Disord, 48(2):583-591. 
21- Schreck, K, A., \& Williams, K.(2005). Food preferences and factors influencing food selectivity for children with autism spectrum disorders, Res Dev Disabil, (4), 353-63.

22- Seiverling, L, Williams, K. E, Ward-Horner, W. J, Sturmey, P. (2011). Interventions to Treat Feeding Problems in Children with Autism Spectrum Disorders: A Comprehensive Review, In Matson, J. L \& Sturmey. P (Eds) International Handbook of Autism and Pervasive

23- Developmental Disorders, 491-508, New York: Springer.

24- Seiverling, L, Williams, K, Sturmey, P, Hart, S.(201 r).Effects of behavioral skills training on parental treatment of childerns food selectivity, Journal of applied behavior analysis, 45, 197-203.

25- Sharp, W. G., Jaquess, D. L. (2009). Bite size and texture assessments to prescribe treatment for severe food selectivity in autism. Behavioral Interventions, 24, 157-170.

26- Strickland, E (2009). Eeating for autism" The 10-step nutrition plan to help treat your child's Autism, Asperger's, or ADHD, UN: Da Capo Lifelong Books.

27- Suarez, M. A (2014). Multicomponent Treatment for Food Selectivity.

28- in Children: Description and Case Report, Nutrition in Clinical Practice, 30 (3), 425 - 431.

29- Suarez, M. A., \& Crinion, K. M. (2015). Food choices of children

30- with autism spectrum disorders. International Journal of School Health, 2 (3), 1-5.

31- Suarez, M, A, Nelson N, W., \& Curtis A, B. (2012). Associations of physiological factors, age, and sensory over-responsivity with food selectivity in children with autism spectrum disorders. Online journal of Occupational Therapy, 1 (1).

32- Tarbox, J., Bermudez, T.L. (2017). Treating Feeding Challenges in Autism: Academic Press

33- Williams, P. G., Dalrymple, N., \& Neal, J. (2000). Eating habits of children with autism.Pediatric Nursing, 26, (3), 259-264.

34- Zimmer, M.H., Hart L.C., Murray, D, S., Bing, N, M., Summer, S. (2012). Food varietyas a predictor of nutritional status among children with autism. Journal of Autism and Developmental Disorders, 42: 549-556. 